ANNALES

UNIVERSITATIS MARIAE CURIE-SKŁODOWSKA

LUBLIN - POLONIA

VOL. LXXI, 2

SECTIO AA

2016

\title{
Synthesis, spectral studies and insect antifeedant activities of some 4-substituted 1-naphthacyl bromides and their esters
}

\author{
Ganesamoorthy Thirunarayanan \\ ${ }^{a}$ Department of Chemistry, Annamalai University, \\ Annamalainagar-608 002, India \\ email:ddrgtnarayanan@gmail.com
}

\begin{abstract}
About nine 4-substituted 1-naphthacyl bromides and its esters have been synthesized by greener synthetic method using fly-ash catalyzed water mediated reaction. These acyl bromides and esters have been characterized by their physical constants, Mass, IR and $\mathrm{NMR}$ spectral data. The $\mathrm{C}=\mathrm{O}$ frequencies frequencies $\left(\mathrm{cm}^{-1}\right)$ of existing rotomers have been assigned and correlated with Hammett substituent constants, F, R and Swain-Lupton's parameters. The insect antifeedant activities of the synthesized acyl bromides and esters have been evaluated using $4^{\text {th }}$ instar larvae Achoea Janatha $L$.
\end{abstract}

Keywords: 4-substituted 1-naphthacyl bromides, 4-substituted 1-naphthacyl benzoates, IR\& NMR spectra, correlation analysis, insect antifeedant activities

\section{INTRODUCTION}

Stereoselective, stereospecific and regioselective synthetic methods are important for the synthesis of biologically active carbonyl compounds [1-3] through solvent-free green reactions like bromination [4-6], esterification[7,8] and calixarene complexation [9]. Green solventassisted and solvent-free reactions are useful for the synthesis of numerous organic compounds such as carbonyl compounds, pyrazolines and imines 
[10-13]. Among these reactions esterification and bromination reactions are important for the formation of carbon-bromine and carbon-oxygen bonds in carbonyl compounds $[14,15]$. Many natural organic compounds have methylene fragment in their structure and it is the origin for biological activity. This methylene fragment is useful for the synthesis of cyclic ketones, acylic ketones, esters, flavones and couma-rone derivatives [16-19]. Many reagents like Copper(II)bromide [20], N,N-dimethylformamide [21], 1,4-dioxanebromooxoniumbromide [22], tribromoacetophenone [23], N-bromosaccharin [24], tribromoacetyl-tetrabutylammonium bromides [6], human esoinophils [25], peroxo-Mntetrabutylammoniumbromides [7,8], bromide-bromates [12], acylammonium salts-LDA quenches with bromine [26], benzylic bromides [27], pyridiniumbromide perbromide [28] and pyridinium bromochromate [29] have been utilized for the bromination of organic substrates. The reagents like $\mathrm{Me}_{2} \mathrm{NSO}_{2} \mathrm{Cl}$ and $\mathrm{N}$,N-dimethylamines [30], $\left(\mathrm{CH}_{3} \mathrm{O}\right)_{4} \mathrm{Si}$ tetra-alkylorthosilicates in presence of $\mathrm{Pd}$ [31], $t$-BuOOC$-\mathrm{CuBr}$ [32], $\mathrm{CO}-\mathrm{CH}_{3} \mathrm{OH}-\mathrm{i}-\mathrm{PrNEt}_{2}$ with $\mathrm{Pd}$ [33], TFFH [34], dimethyl formamide-dipropylacetal or dimethylformamide-di-isopropylacetal with pyridine $[14,15], 4-(4,6$-dimethoxy-1,3,5-triazin-2-yl)-4-methyl-morpholiniumchloride, N-methyl-2-bromo pyridinium iodide, N-methyl-2-chloropyridinium iodide and $\mathrm{N}$-methyl-2- bromopyridinium tosylates [35], metal salt in liquid EDTA [36], Lewis acids and bases [37-39] have been employed for esterification of acids, acid chlorides, ethers, alcohols, halo alkanes, alkenes and salt derivatives. Herein the author reports an effective method for bromination selectively at the side chain in a 4-substituted 1-naphthyl ketones with potassium bromide-bromate mixture (Winkler's reagent) in the presence of fly-ash in water medium.

The corresponding esters were synthesized by nucleophilic substitution of carboxylate anion (finally ester formation) of various 4-substituted 1-naphthacylbromides with sodium benzoate in the presence of fly-ash in water medium and the yield obtained was more than $60 \%$. The 4-substituted 1-naphthacyl bromides and their esters exist as different rotamers. These rotamers were identified by their infrared spectral data of $\mathrm{C}=\mathrm{O}$ group absorptions. The observed frequencies have been correlated with Hammett substituent constants. From the regression analysis results, the effect of substituents on the carbonyl carbon atom have been studied. The author also studied the insect antifeedant activities of all the synthesized ketones and esters using the $4^{\text {th }}$ instar larvae Achoea Janatha $L$ with castor leaf discs. 


\section{EXPERIMENTAL}

\subsection{General}

Fly ash was obtained from Thermal Power Plant-II, Neyveli Lignite Corporation (NLC), Neyveli, Tamil Nadu, India. All chemicals used were purchased from Sigma-Aldrich and E-Merck Chemical Companies. Melting points of all acyl bromides and esters were determined in open glass capillaries on Mettler FP51 melting point apparatus and are uncorrected. Infrared spectra $\left(\mathrm{KBr}, 4000-400 \mathrm{~cm}^{-1}\right)$ were recorded on Avatar-300 Fourier transform spectrophotometer. The NMR spectra of all compounds were recorded in Bruker AV500 spectrometer operating at $500 \mathrm{MHz}$ for ${ }^{1} \mathrm{H}$ NMR spectra and $125 \mathrm{MHz}$ for ${ }^{13} \mathrm{C}$ NMR spectra in $\mathrm{CDCl}_{3}$ solvent using TMS as internal standard. Electron impact (EI) $(70 \mathrm{eV})$ and chemical ionization mode $\mathrm{FAB}^{+}$mass spectra have been recorded with a JEOL JMS600H spectrometer.

\subsubsection{General procedure for bromination of 4-substituted 1-naphthyl methyl ketones}

In a $100 \mathrm{~cm}^{3}$ flask, 4-substituted 1-naphthyl methyl ketones $(4.16 \mathrm{mmol})$ in ethanol $\left(20 \mathrm{~cm}^{3}\right), 0.5 \mathrm{~g}$ of fly ash and $10 \mathrm{~cm}^{3}$ of water were stirred on magnetic stirrer. To this mixture, $10 \mathrm{~cm}^{3}$ of Winkler's solution (Bromate-bromide solution) was added drop-wise with stirring for 45 min. until a decolorization of orange colour [40] (Scheme 1).

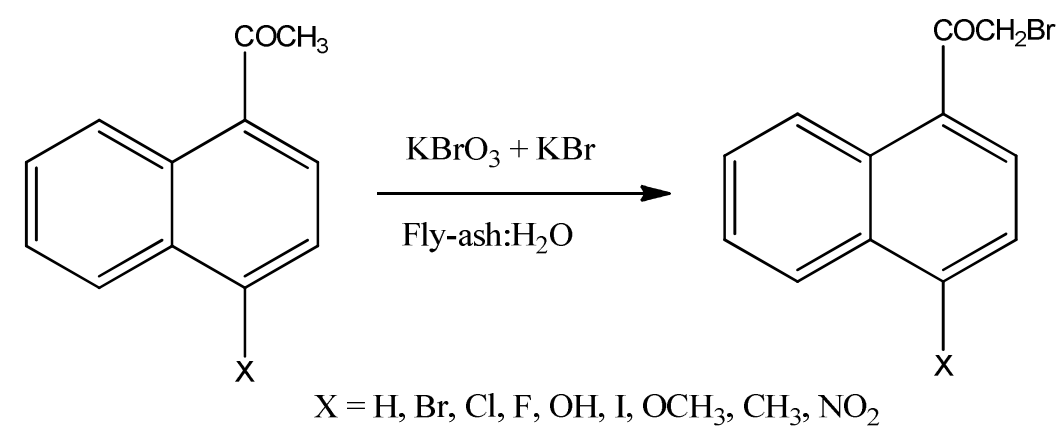

Scheme 1. Synthesis of 4-substituted 1-naphathaxyl bromides.

Thin layer chromatography was used to monitor the reaction. After completion of the reaction, the fly-ash was separated by simple filtration. The organic extract was cooled in an ice bath to give naphthacyl 
bromides, filtered at the vacuum pump and dried. Further the crude naphthacyl bromides were purified by column chromatography using ethyl acetate-dichloromethane (6:4) eluents. The yield, physical constants and spectroscopic data of all synthesized 4-substituted 1-naphthacylbromides (1-9) are summarized below.

1-Naphthacyl bromide (1). Yield: 67\%, m.p. 92-93 ${ }^{\circ} \mathrm{C}$, IR(4000-400, $\left.\mathrm{KBr}, \mathrm{cm}^{-1}\right) v=1667\left(\mathrm{CO}_{\text {cis }}\right), 1664\left(\mathrm{CO}_{\text {gauche }}\right), 648(\mathrm{C}-\mathrm{Br}), 2998\left(\mathrm{C}-\mathrm{H}_{\text {alip. }}\right)$, $3021\left(\mathrm{C}-\mathrm{H}_{\text {aro. }}\right) ; 1 \mathrm{H} \mathrm{NMR}\left(\mathrm{CDCl}_{3}, 500 \mathrm{MHz}, \mathrm{ppm}\right) \delta=4.224\left(\mathrm{~s}, 2 \mathrm{H},-\mathrm{CH}_{2}\right)$, 7.202-8.781(m, 7H, $\mathrm{Ar}-\mathrm{H}) ;{ }^{13} \mathrm{C} \quad \mathrm{NMR}\left(\mathrm{CDCl}_{3}, 125 \mathrm{MHz}, \quad \mathrm{ppm}\right)$ $\delta=187.38(\mathrm{CO}), 32.37\left(\mathrm{CH}_{2}\right), 124.35-135.73(\mathrm{Ar}-\mathrm{C}) ; \mathrm{M} . \mathrm{F} . \mathrm{C}_{12} \mathrm{H}_{9} \mathrm{BrO}$, M.W. 248; Mass $(\mathrm{m} / \mathrm{z})=248\left[\mathrm{M}^{+}\right], 250\left[\mathrm{M}^{+2}\right], 169,155,151,121,107$, $93,42,42,14$.

4-Bromo-1-naphthacyl bromide (2). Yield: $62 \%$, m.p. $117-118^{\circ} \mathrm{C}$, $\operatorname{IR}\left(4000-400, \mathrm{KBr}, \mathrm{cm}^{-1}\right) \quad v=1669\left(\mathrm{CO}_{\text {cis }}\right), \quad 1662\left(\mathrm{CO}_{\text {gauche }}\right), \quad 657$,

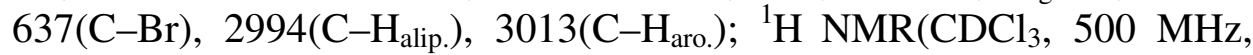
ppm) $\delta=4.476\left(\mathrm{~s}, \quad 2 \mathrm{H}, \quad-\mathrm{CH}_{2}\right), \quad 7.651-8.972(\mathrm{~m}, \quad 6 \mathrm{H}, \quad \mathrm{Ar}-\mathrm{H})$; ${ }^{13} \mathrm{CNMR}\left(\mathrm{CDCl}_{3}, 125 \mathrm{MHz}, \mathrm{ppm}\right) \delta=187.67(\mathrm{CO}), 33.78\left(\mathrm{CH}_{2}\right), 125.81-$ 132.67(Ar-C); M. F. $\mathrm{C}_{12} \mathrm{H}_{8} \mathrm{Br}_{2} \mathrm{O}$, M.W. 326; Mass $(\mathrm{m} / \mathrm{z})=326\left[\mathrm{M}^{+}\right]$, $328\left[\mathrm{M}^{+2}\right], 330\left[\mathrm{M}^{+4}\right], 247,233,205,153,107,92,77$.

4-Chloro-1-naphthacyl bromide (3). Yield: 65\%, m.p. $123-124^{\circ} \mathrm{C}$, $\operatorname{IR}\left(4000-400, \mathrm{KBr}, \mathrm{cm}^{-1}\right) v=1671\left(\mathrm{CO}_{\text {cis }}\right), 1666\left(\mathrm{CO}_{\text {gauche }}\right), 637(\mathrm{C}-\mathrm{Br})$,

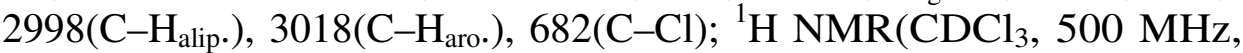
ppm) $\delta=3.571\left(\mathrm{~s}, 2 \mathrm{H},-\mathrm{CH}_{2}\right), 7.510-8.182(\mathrm{~m}, 6 \mathrm{H}, \mathrm{Ar}-\mathrm{H}) ;{ }^{13} \mathrm{C}$ $\operatorname{NMR}\left(\mathrm{CDCl}_{3}, 125 \mathrm{MHz}, \mathrm{ppm}\right) \delta=186.75(\mathrm{CO}), 30.76\left(\mathrm{CH}_{2}\right), 124.32$ 135.76(Ar-C); M. F. $\mathrm{C}_{12} \mathrm{H}_{8} \mathrm{BrClO}$, M.W. 282; Mass $(\mathrm{m} / \mathrm{z})=282\left[\mathrm{M}^{+}\right]$, $284\left[\mathrm{M}^{+2}\right], 286\left[\mathrm{M}^{+4}\right], 207,193,165,141,107,92,77,35$.

4-Fluoro-1-naphthacyl bromide (4). Yield: 68\%, m.p. 92-93 $\mathrm{C}$, IR(4000$\left.400, \mathrm{KBr}, \mathrm{cm}^{-1}\right) v=1676\left(\mathrm{CO}_{\text {cis }}\right), 1664\left(\mathrm{CO}_{\text {gauche }}\right), 648(\mathrm{C}-\mathrm{Br}), 2997(\mathrm{C}-$ Halip.), 3014(C-Haro.), 613(C-F); ${ }^{1} \mathrm{H} \mathrm{NMR}\left(\mathrm{CDCl}_{3}, 500 \mathrm{MHz}, \mathrm{ppm}\right) \delta=$ 4.384(s, $\left.\quad 2 \mathrm{H}, \quad-\mathrm{CH}_{2}\right), \quad 7.021-8.134(\mathrm{~m}, \quad 6 \mathrm{H}, \quad \mathrm{Ar}-\mathrm{H}) ; \quad{ }^{13} \mathrm{C}$ $\operatorname{NMR}\left(\mathrm{CDCl}_{3}, 125 \mathrm{MHz}, \mathrm{ppm}\right) \delta=183.62(\mathrm{CO}), 32.45\left(\mathrm{CH}_{2}\right), 110.72$ 166.73(Ar-C); M. F. $\mathrm{C}_{12} \mathrm{H}_{8} \mathrm{BrFO}$, M.W. 266; Mass $(\mathrm{m} / \mathrm{z})=266\left[\mathrm{M}^{+}\right]$, $268\left[\mathrm{M}^{+2}\right], 270\left[\mathrm{M}^{+4}\right], 247,187,173,145,107,95,71,19$.

4-Hydroxy-1-naphthacyl bromide (5). Yield: $64 \%$, m.p. $103-104^{\circ} \mathrm{C}$, $\operatorname{IR}\left(4000-400, \mathrm{KBr}, \mathrm{cm}^{-1}\right) v=1664\left(\mathrm{CO}_{\text {cis }}\right), 1660\left(\mathrm{CO}_{\text {gauche }}\right), 628(\mathrm{C}-\mathrm{Br})$,

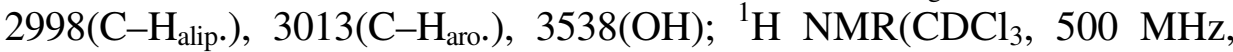
ppm) $\delta=4.282\left(\mathrm{~s}, \quad 2 \mathrm{H},-\mathrm{CH}_{2}\right), 6.623-8.781(\mathrm{~m}, \quad 6 \mathrm{H}, \quad \mathrm{Ar}-\mathrm{H}) ;{ }^{13} \mathrm{C}$ 
$\operatorname{NMR}\left(\mathrm{CDCl}_{3}, 125 \mathrm{MHz}, \mathrm{ppm}\right) \delta=187.32(\mathrm{CO}), 33.23\left(\mathrm{CH}_{2}\right), 110.52-$ 132.87(Ar-C); M. F. $\mathrm{C}_{12} \mathrm{H}_{9} \mathrm{BrO}_{2}$, M.W. 264; Mass $(\mathrm{m} / \mathrm{z})=264\left[\mathrm{M}^{+}\right]$, 266[ $\left.\mathrm{M}^{+2}\right], 187,171,154,107,91,71,52,28$.

4-Iodo-1-naphthacyl bromide (6). Yield: $65 \%$, m.p. $114-115^{\circ} \mathrm{C}$, IR(4000$\left.400, \mathrm{KBr}, \mathrm{cm}^{-1}\right) v=1679\left(\mathrm{CO}_{\text {cis }}\right), 1668\left(\mathrm{CO}_{\text {gauche }}\right), 653(\mathrm{C}-\mathrm{Br}), 2997(\mathrm{C}-$ $\left.\mathrm{H}_{\text {alip. }}\right), 3016\left(\mathrm{C}-\mathrm{H}_{\text {aro }}\right), 638(\mathrm{C}-\mathrm{I}) ;{ }^{1} \mathrm{H} \mathrm{NMR}\left(\mathrm{CDCl}_{3}, 500 \mathrm{MHz}, \mathrm{ppm}\right) \delta=$ 4.471(s, $\left.2 \mathrm{H},-\mathrm{CH}_{2}\right), 7.271-8.573(\mathrm{~m}, 6 \mathrm{H}, \mathrm{Ar}-\mathrm{H}) ;{ }^{13} \mathrm{C} \mathrm{NMR}\left(\mathrm{CDCl}_{3}, 125\right.$ $\mathrm{MHz}, \mathrm{ppm}) \delta=184.43(\mathrm{CO}), 33.43\left(\mathrm{CH}_{2}\right), 104.35-141.02(\mathrm{Ar}-\mathrm{C})$; M. F. $\mathrm{C}_{12} \mathrm{H}_{8}$ BrIO, M.W. 375; Mass $(\mathrm{m} / \mathrm{z})=375\left[\mathrm{M}^{+}\right], 377\left[\mathrm{M}^{+2}\right], 379\left[\mathrm{M}^{+4}\right], 323$, 281, 253, 249, 247, 187, 127, 121, 107, 92, 78.

4-Methoxy-1-naphthacyl bromide (7). Yield: $67 \%$, m.p. $107-108^{\circ} \mathrm{C}$, $\operatorname{IR}\left(4000-400, \mathrm{KBr}, \mathrm{cm}^{-1}\right) v=1674\left(\mathrm{CO}_{\text {cis }}\right), 1670\left(\mathrm{CO}_{\text {gauche }}\right), 639(\mathrm{C}-\mathrm{Br})$, $2995\left(\mathrm{C}-\mathrm{H}_{\text {alip. }}\right), \quad 3025\left(\mathrm{C}-\mathrm{H}_{\text {aro. }}\right), \quad 1021(\mathrm{C}-\mathrm{O}-\mathrm{C}) ;{ }^{1} \mathrm{H} \mathrm{NMR}\left(\mathrm{CDCl}_{3}, 500\right.$ $\mathrm{MHz}, \mathrm{ppm}) \delta=4.662\left(\mathrm{~s}, 2 \mathrm{H},-\mathrm{CH}_{2}\right), 7.254-8.812(\mathrm{~m}, 6 \mathrm{H}, \mathrm{Ar}-\mathrm{H})$, $3.671\left(\mathrm{~s}, 3 \mathrm{H},-\mathrm{OCH}_{3}\right) ;{ }^{13} \mathrm{C} \mathrm{NMR}\left(\mathrm{CDCl}_{3}, 125 \mathrm{MHz}, \mathrm{ppm}\right) \delta=183.92(\mathrm{CO})$, 32.74( $\left(\mathrm{CH}_{2}\right)$, 105.28-162.772( $\left.\mathrm{Ar}-\mathrm{C}\right), 63.71\left(-\mathrm{OCH}_{3}\right) ;$ M. F. $\mathrm{C}_{13} \mathrm{H}_{11} \mathrm{BrO}_{2}$, M.W. 278; Mass $(\mathrm{m} / \mathrm{z})=278\left[\mathrm{M}^{+}\right], 279\left[\mathrm{M}^{+1}\right], 280\left[\mathrm{M}^{+2}\right], 247,199,185$, 157, 118, 107, 91, 77, 40, 28, 15.

4-Methyl-1-naphthacyl bromide (8). Yield: 65\%, m.p. 96-97 $\mathrm{C}$, IR(4000$\left.400, \mathrm{KBr}, \mathrm{cm}^{-1}\right) v=1668\left(\mathrm{CO}_{\text {cis }}\right), 1665\left(\mathrm{CO}_{\text {gauche }}\right), 627(\mathrm{C}-\mathrm{Br}), 2998(\mathrm{C}-$ $\left.\mathrm{H}_{\text {alip. }}\right), \quad 3019\left(\mathrm{C}-\mathrm{H}_{\text {aro. }}\right) ; \quad{ }^{1} \mathrm{H} \quad \mathrm{NMR}\left(\mathrm{CDCl}_{3}, \quad 500 \mathrm{MHz}, \quad \mathrm{ppm}\right)$ $\delta=4.383\left(\mathrm{~s}, 2 \mathrm{H},-\mathrm{CH}_{2}\right), 7.052-8.027(\mathrm{~m}, 6 \mathrm{H}, \mathrm{Ar}-\mathrm{H}), 3.921\left(\mathrm{~s}, 3 \mathrm{H},-\mathrm{CH}_{3}\right)$; ${ }^{13} \mathrm{C} \mathrm{NMR}\left(\mathrm{CDCl}_{3}, 125 \mathrm{MHz}, \mathrm{ppm}\right) \delta=187.86(\mathrm{CO}), 33.17\left(\mathrm{CH}_{2}\right), 123.81-$ 140.01(Ar-C), 26.75(- $\left.\mathrm{CH}_{3}\right)$; M. F. $\mathrm{C}_{13} \mathrm{H}_{11} \mathrm{BrO}$, M.W. 262; Mass $(\mathrm{m} / \mathrm{z})$ $=262\left[\mathrm{M}^{+}\right], 268\left[\mathrm{M}^{+2}\right], 247,184,169,141,126,107,91,77,15$.

4-Nitro-1-naphthacyl bromide (9). Yield: 63\%, m.p. 131-132 ${ }^{\circ} \mathrm{C}$, IR(4000$\left.400, \mathrm{KBr}, \mathrm{cm}^{-1}\right) v=1683\left(\mathrm{CO}_{\text {cis }}\right), 1676\left(\mathrm{CO}_{\text {gauche }}\right), 658(\mathrm{C}-\mathrm{Br}), 2998(\mathrm{C}-$ $\left.\mathrm{H}_{\text {alip. }}\right), \quad 3025\left(\mathrm{C}-\mathrm{H}_{\text {aro. }}\right) ; \quad{ }^{1} \mathrm{H} \quad \mathrm{NMR}\left(\mathrm{CDCl}_{3}, \quad 500 \mathrm{MHz}, \quad \mathrm{ppm}\right)$ $\delta=4.731\left(\mathrm{~s}, 2 \mathrm{H},-\mathrm{CH}_{2}\right), 7.517-9.187(\mathrm{~m}, 6 \mathrm{H}, \mathrm{Ar}-\mathrm{H}) ;{ }^{13} \mathrm{C} \mathrm{NMR}\left(\mathrm{CDCl}_{3}\right.$, $125 \mathrm{MHz}, \mathrm{ppm}) \delta=187.33(\mathrm{CO}), 33.75\left(\mathrm{CH}_{2}\right), 123.33-151.52(\mathrm{Ar}-\mathrm{C})$; M. F. $\mathrm{C}_{12} \mathrm{H}_{8} \mathrm{BrNO}_{3}$, M.W. 294; Mass $(\mathrm{m} / \mathrm{z})=294\left[\mathrm{M}^{+}\right], 296\left[\mathrm{M}^{+2}\right], 247,214$, 200, 198, 172, 121, 107, 93, 77, 46, 54.

2.1.2. General procedure for the synthesis of 4-substituted 1-naphthacylbenzoates

A solution of 4-substituted 1-naphthacylbromides $(4.23 \mathrm{mmol})$ in $50 \%$ ethanol $\left(15 \mathrm{~cm}^{3}\right)$, sodium benzoate $(4.15 \mathrm{mmol})$ in $50 \%$ ethanol 
$\left(15 \mathrm{~cm}^{3}\right)$ and $0.5 \mathrm{~g}$ of fly ash were refluxed [40] for $4 \mathrm{~h}$ (Scheme 2). Thin layer chromatography was used to monitor the reaction. After completion of the reaction the whole mass was allowed to remain undisturbed overnight. The fly-ash was separated by filtration. The organic layer was extracted with ether and concentrated to a small volume.

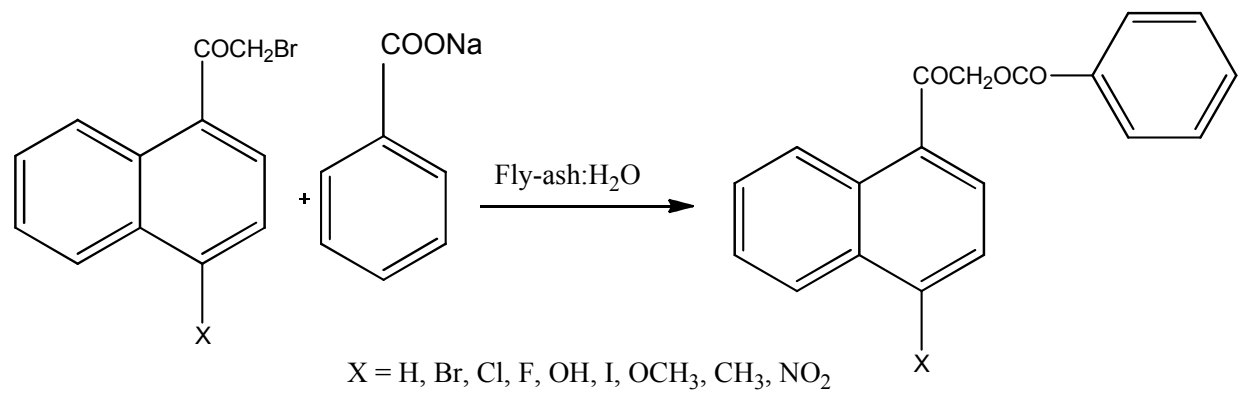

Scheme 2. Synthesis of 4-substituted 1-naphthacyl benzoates.

The precipitated ester was filtered off and purified by recrystallization from methanol to give more than $70 \%$ product. The analytical and spectral data of all the synthesized 4-substituted-1-naphthacyl benzoates (10-18) are presented below.

1-Naphathacyl benzoate (10). Yield: $67 \%$, m.p. $122-123^{\circ} \mathrm{C}$, IR(4000$\left.-400, \mathrm{KBr}, \mathrm{cm}^{-1}\right) v=1738\left(\right.$ Keto $\left.\mathrm{CO}_{\text {cis }}\right), 1701$ (Keto $\left.\mathrm{CO}_{\text {gauche }}\right)$, 1615(Ester $\mathrm{CO}_{\text {cis }}$ ), 1583(Ester $\left.\mathrm{CO}_{\text {gauche }}\right), 2999\left(\mathrm{C}-\mathrm{H}_{\text {alip }}\right.$ ), 3016(CHaro.); $1 \mathrm{H} \mathrm{NMR}\left(\mathrm{CDCl}_{3}, 500 \mathrm{MHz}, \mathrm{ppm}\right) \delta=5.316(\mathrm{~s}, 2 \mathrm{H},-\mathrm{CH} 2)$, 7.326-8.675(m, 12H, $\mathrm{Ar}-\mathrm{H}) ; 13 \mathrm{C} \mathrm{NMR}\left(\mathrm{CDCl}_{3}, 125 \mathrm{MHz}, \mathrm{ppm}\right)$ $\delta=187.38($ Keto $\mathrm{CO}), 68.23\left(\mathrm{CH}_{2}\right), 168.31($ Ester CO), 123.98-134.47(Ar-C); M. F. $\mathrm{C}_{19} \mathrm{H}_{14} \mathrm{O}_{3}$, M.W. 290; Mass (m/z) = 290[M+], 282, 186, 169, 155, 127, 122, 107, 93, 77, 28.

4-Bromo-1-naphathacyl benzoate (11). Yield: 63\%, m.p. $113-114^{\circ} \mathrm{C}$, $\operatorname{IR}\left(4000-400, \mathrm{KBr}, \mathrm{cm}^{-1}\right) v=1742$ (Keto $\left.\mathrm{CO}_{\text {cis }}\right), 1713$ (Keto $\mathrm{CO}_{\text {gauche }}$ ), 1654(Ester $\mathrm{CO}_{\text {cis }}$ ), 1638(Ester $\mathrm{CO}_{\text {gauche }}$ ), 2991(C-Halip.), 3015(C-Haro.); $1 \mathrm{H} \mathrm{NMR}\left(\mathrm{CDCl}_{3}, 500 \mathrm{MHz}, \mathrm{ppm}\right) \delta=5.274\left(\mathrm{~s}, 2 \mathrm{H},-\mathrm{CH}_{2}\right)$, 7.381-8.102(m, $11 \mathrm{H}, \mathrm{Ar}-\mathrm{H}) ;{ }^{13} \mathrm{C} \mathrm{NMR}\left(\mathrm{CDCl}_{3}, 125 \mathrm{MHz}, \mathrm{ppm}\right)$ $\delta=187.68($ Keto $\mathrm{CO}), \quad 63.68\left(\mathrm{CH}_{2}\right)$, 169.79(Ester $\left.\mathrm{CO}\right), 126.81-$ 135.29(Ar-C); M. F. C19H13BrO3, M.W. 368; Mass $(\mathrm{m} / \mathrm{z})=368\left[\mathrm{M}^{+}\right]$, $370\left[\mathrm{M}^{+2}\right], 292,262,246,163,137,121,105,91,77,51,28$. 
4-Bromo-1-naphathacyl benzoate (12). Yield: $62 \%$, m.p. $181-182^{\circ} \mathrm{C}$, $\operatorname{IR}\left(4000-400, \mathrm{KBr}, \mathrm{cm}^{-1}\right) v=1742\left(\right.$ Keto $\left.\mathrm{CO}_{\text {cis }}\right), 1702$ (Keto $\mathrm{CO}_{\text {gauche }}$ ), 1643(Ester $\mathrm{CO}_{\text {cis }}$ ), 1602(Ester $\left.\mathrm{CO}_{\text {gauche }}\right), 2994\left(\mathrm{C}-\mathrm{H}_{\text {alip. }}\right)$, 3028(C-Haro.); ${ }^{1} \mathrm{H} \mathrm{NMR}(\mathrm{CDCl} 3,500 \mathrm{MHz}, \mathrm{ppm}) \delta=5.834\left(\mathrm{~s}, 2 \mathrm{H},-\mathrm{CH}_{2}\right), 7.382-$ 7.912(m, $11 \mathrm{H}, \quad \mathrm{Ar}-\mathrm{H}) ;{ }^{13} \mathrm{C} \quad \mathrm{NMR}\left(\mathrm{CDCl}_{3}, \quad 125 \mathrm{MHz}, \quad \mathrm{ppm}\right)$ $\delta=186.35($ Keto $\mathrm{CO}), 62.68\left(\mathrm{CH}_{2}\right), 168.45$ (Ester $\left.\mathrm{CO}\right), 123.82-$ 136.71(Ar-C); M. F. $\mathrm{C}_{19} \mathrm{H}_{13} \mathrm{ClO}_{3}$, M.W. 324; Mass $(\mathrm{m} / \mathrm{z})=324\left[\mathrm{M}^{+}\right]$, 326[ $\left.\mathrm{M}^{+2}\right], 296,247,219,202,190,162,107,93,91,77,28$.

4-Fluoro-1-naphathacyl benzoate (13). Yield: $68 \%$, m.p. $147-148^{\circ} \mathrm{C}$, $\operatorname{IR}\left(4000-400, \mathrm{KBr}, \mathrm{cm}^{-1}\right) v=1740\left(\right.$ Keto $\left.\mathrm{CO}_{\text {cis }}\right), 1705$ (Keto $\left.\mathrm{CO}_{\text {gauche }}\right)$, 1645(Ester COcis), 1605(Ester $\left.\mathrm{CO}_{\text {gauche }}\right), 2995\left(\mathrm{C}-\mathrm{H}_{\text {alip. }}\right.$ ), 3016(C- $\left.\mathrm{H}_{\text {aro. }}\right)$; ${ }^{1} \mathrm{H} \mathrm{NMR}\left(\mathrm{CDCl}_{3}, 500 \mathrm{MHz}, \mathrm{ppm}\right) \delta=5.274\left(\mathrm{~s}, 2 \mathrm{H},-\mathrm{CH}_{2}\right), 7.323-$ $8.014(\mathrm{~m}, \quad 11 \mathrm{H}, \quad \mathrm{Ar}-\mathrm{H}) ;{ }^{13} \mathrm{C} \quad \mathrm{NMR}\left(\mathrm{CDCl}_{3}, \quad 125 \mathrm{MHz}, \quad \mathrm{ppm}\right)$ $\delta=184.47($ Keto $\mathrm{CO}), \quad 67.28\left(\mathrm{CH}_{2}\right), \quad 167.34($ Ester $\mathrm{CO})$, 109.74136.72(Ar-C); M. F. $\mathrm{C}_{19} \mathrm{H}_{13} \mathrm{FO}_{3}$, M.W. 308; Mass $(\mathrm{m} / \mathrm{z})=308\left[\mathrm{M}^{+}\right]$, 310[ $\left.\mathrm{M}^{+2}\right], 231,247,219,203,173,145,121,109,105,77,28$.

4-Hydroxy-1-naphathacyl benzoate (14). Yield: 64\%, m.p. $111-112^{\circ} \mathrm{C}$, $\operatorname{IR}\left(4000-400, \mathrm{KBr}, \mathrm{cm}^{-1}\right) v=1664\left(\right.$ Keto $\left.\mathrm{CO}_{\text {cis }}\right), 1725\left(\right.$ Keto $\left.\mathrm{CO}_{\text {gauche }}\right)$,

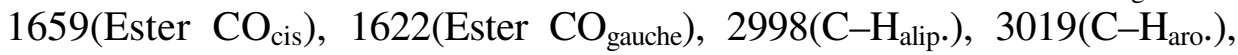
$3544(\mathrm{OH}) ; 1 \mathrm{H} \mathrm{NMR}\left(\mathrm{CDCl}_{3}, 500 \mathrm{MHz}, \mathrm{ppm}\right) \delta=5.325\left(\mathrm{~s}, 2 \mathrm{H}-\mathrm{CH}_{2}\right)$, 6.825-7.924(m, $11 \mathrm{H}, \quad \mathrm{Ar}-\mathrm{H}) ;{ }^{13} \mathrm{C} \operatorname{NMR}\left(\mathrm{CDCl}_{3}, 125 \mathrm{MHz}, \mathrm{ppm}\right)$ $\delta=187.72($ Keto $\mathrm{CO}), \quad 62.61\left(\mathrm{CH}_{2}\right)$, 168.14(Ester $\left.\mathrm{CO}\right)$, 108.82164.75(Ar-C); M. F. $\mathrm{C}_{19} \mathrm{H}_{14} \mathrm{O}_{4}$, M.W. 352; Mass (m/z) $=352\left[\mathrm{M}^{+}\right], 290$, 289, 227, 186, 171, 163, 143, 135, 121, 107, 105, 91, 17.

4-Iodo-1-naphathacyl benzoate (15). Yield: 64\%, m.p. $127-128^{\circ} \mathrm{C}$, $\operatorname{IR}\left(4000-400, \mathrm{KBr}, \mathrm{cm}^{-1}\right) v=1746\left(\right.$ Keto $\left.\mathrm{CO}_{\text {cis }}\right), 1714\left(\right.$ Keto $\left.\mathrm{CO}_{\text {gauche }}\right)$, 1650(Ester $\left.\mathrm{CO}_{\text {cis }}\right), 1637$ (Ester $\left.\mathrm{CO}_{\text {gauche }}\right), 2998\left(\mathrm{C}-\mathrm{H}_{\text {alip. }}\right.$ ), 3025(C- $\left.\mathrm{H}_{\text {aro. }}\right)$; ${ }^{1} \mathrm{H} \mathrm{NMR}\left(\mathrm{CDCl}_{3}, 500 \mathrm{MHz}, \mathrm{ppm}\right) \delta=4.832\left(\mathrm{~s}, 2 \mathrm{H},-\mathrm{CH}_{2}\right), 7.328-$ $8.051(\mathrm{~m}, \quad 11 \mathrm{H}, \quad \mathrm{Ar}-\mathrm{H}) ; \quad{ }^{13} \mathrm{C} \quad \mathrm{NMR}\left(\mathrm{CDCl}_{3}, \quad 125 \mathrm{MHz}, \quad \mathrm{ppm}\right)$ $\delta=187.74($ Keto $\mathrm{CO})$, 63.62 $\left(\mathrm{CH}_{2}\right)$, 169.69(Ester CO), 105.75136.32(Ar-C); M. F. C19H13IO3, M.W. 416; Mass $(\mathrm{m} / \mathrm{z})=416\left[\mathrm{M}^{+}\right]$, $418\left[\mathrm{M}^{+2}\right], 339,311,295,281,253,163,145,135,127,121,105,77$.

4-Methoxy-1-naphathacyl benzoate (16). Yield: $61 \%$, m.p. $116-117^{\circ} \mathrm{C}$, $\operatorname{IR}\left(4000-400, \mathrm{KBr}, \mathrm{cm}^{-1}\right) v=1755\left(\right.$ Keto $\left.\mathrm{CO}_{\text {cis }}\right), 1713$ (Keto $\left.\mathrm{CO}_{\text {gauche }}\right)$, 1646(Ester $\left.\mathrm{CO}_{\text {cis }}\right), 1632$ (Ester $\left.\mathrm{CO}_{\text {gauche }}\right), 2999\left(\mathrm{C}-\mathrm{H}_{\text {alip. }}\right)$, 3016(C-H aro. $)$, $1025(\mathrm{C}-\mathrm{O}-\mathrm{C}) ;{ }^{1} \mathrm{H} \mathrm{NMR}\left(\mathrm{CDCl}_{3}, 500 \mathrm{MHz}, \mathrm{ppm}\right) \delta=4.835(\mathrm{~s}, 2 \mathrm{H},-$ $\left.\mathrm{CH}_{2}\right), \quad 7.361-7.921(\mathrm{~m}, \quad 11 \mathrm{H}, \quad \mathrm{Ar}-\mathrm{H}), \quad 3.435\left(\mathrm{~s}, \quad 3 \mathrm{H}, \quad \mathrm{OCH}_{3}\right) ;{ }^{13} \mathrm{C}$ $\mathrm{NMR}\left(\mathrm{CDCl}_{3}, 125 \mathrm{MHz}, \mathrm{ppm}\right) \delta=183.19$ (Keto $\left.\mathrm{CO}\right), 64.57(\mathrm{CH} 2)$, 
165.32(Ester CO), 105.62-162.14(Ar-C), $57.65\left(\mathrm{OCH}_{3}\right) ; \quad$ M. F. $\mathrm{C}_{20} \mathrm{H}_{16} \mathrm{O}_{4}$, M.W. 320; Mass $(\mathrm{m} / \mathrm{z})=320\left[\mathrm{M}^{+}\right], 264,243,215,199,185$, 163, 157, 135, 121, 107, 105, 93, 91, 77, 56, 15.

4-Methyl-1-naphathacyl benzoate (17). Yield: $67 \%$, m.p. $131-132^{\circ} \mathrm{C}$, $\operatorname{IR}\left(4000-400, \mathrm{KBr}, \mathrm{cm}^{-1}\right) v=1721\left(\right.$ Keto $\left.\mathrm{CO}_{\text {cis }}\right), 1706\left(\right.$ Keto $\left.\mathrm{CO}_{\text {gauche }}\right)$,

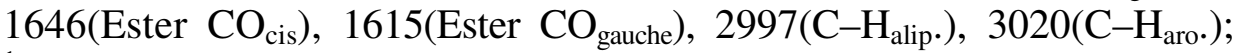
${ }^{1} \mathrm{H} \mathrm{NMR}\left(\mathrm{CDCl}_{3}, 500 \mathrm{MHz}, \mathrm{ppm}\right) \delta=4.621\left(\mathrm{~s}, 2 \mathrm{H},-\mathrm{CH}_{2}\right), 7.051-$ 7.813(m, $11 \mathrm{H}, \mathrm{Ar}-\mathrm{H}), 2.384\left(\mathrm{~s}, 3 \mathrm{H}, \mathrm{CH}_{3}\right) ;{ }^{13} \mathrm{C} \mathrm{NMR}\left(\mathrm{CDCl}_{3}, 125 \mathrm{MHz}\right.$, ppm) $\delta=186.37($ Keto $\mathrm{CO}), 63.68\left(\mathrm{CH}_{2}\right), 166.42($ Ester $\mathrm{CO}), 122.08-$ 141.38(Ar-C), 26.32( $\left(\mathrm{CH}_{3}\right)$; M. F. $\mathrm{C}_{20} \mathrm{H}_{16} \mathrm{O}_{3}$, M.W. 304; Mass $(\mathrm{m} / \mathrm{z})$ $=304\left[\mathrm{M}^{+}\right], 228,215,200,185,170,163,157,142,135,121,107,105$, 93, 91, 52, 28.

4-Nitro-1-naphathacyl benzoate (18). Yield: $63 \%$, m.p. $121-122^{\circ} \mathrm{C}$, $\operatorname{IR}\left(4000-400, \mathrm{KBr}, \mathrm{cm}^{-1}\right) v=1747\left(\right.$ Keto $\left.\mathrm{CO}_{\text {cis }}\right), 1716\left(\right.$ Keto $\left.\mathrm{CO}_{\text {gauche }}\right)$, $1650\left(\right.$ Ester $\left.\mathrm{CO}_{\text {cis }}\right), 1635$ (Ester $\left.\mathrm{CO}_{\text {gauche }}\right), 2999\left(\mathrm{C}-\mathrm{H}_{\text {alip }}.\right), 3022\left(\mathrm{C}-\mathrm{H}_{\text {aro. }}\right)$; ${ }^{1} \mathrm{H} \mathrm{NMR}\left(\mathrm{CDCl}_{3}, 500 \mathrm{MHz}, \mathrm{ppm}\right) \delta=4.826\left(\mathrm{~s}, 2 \mathrm{H},-\mathrm{CH}_{2}\right), 7.381-7.314$ $(\mathrm{m}, 11 \mathrm{H}, \mathrm{Ar}-\mathrm{H}) ;{ }^{13} \mathrm{C} \mathrm{NMR}\left(\mathrm{CDCl}_{3}, 125 \mathrm{MHz}, \mathrm{ppm}\right) \delta=187.72$ (Keto $\mathrm{CO})$, 65.36( $\left.\mathrm{CH}_{2}\right)$, 169.79(Ester CO), 104.31-136.83(Ar-C); M. F. $\mathrm{C}_{19} \mathrm{H}_{13} \mathrm{NO}_{5}$, M.W. 335; Mass $(\mathrm{m} / \mathrm{z})=335\left[\mathrm{M}^{+}\right], 289,258,230,214,200$, 185, 172, 169, 163, 155, 135, 127, 121, 105, 77, 46, 44.

\section{RESULTS AND DISCUSSION}

Many solvent-assisted brominating methods for the bromination of alkyl and aryl compounds by direct use of bromine solution in alkaline or acid medium have been reported in the literature [3, 41-43]. In the present work, the author has attempted to brominate naphthyl keto compounds with an alternative reagent like Winkler's solution (bromate-bromide solution) in the presence of aqueous phase catalyst fly-ash. The bromate-bromide solution was harmless to the reaction [3, 41-43] as well as the environment. Various 1-naphthyl ketones containing electron withdrawing and electron donating groups in position 4 have been subjected to bromination at acyl methyl group with Winkler's solution in the presence of fly-ash catalyst under water medium (Scheme 1). The reaction got completed within $1 \mathrm{~h}$ and more than $60 \%$ yields of 4-substituted 1-naphthacyl bromides (1-9) were obtained. In this reaction, no bromination occurs in naphthyl ring. The synthesized 4-substituted 1-naphthacyl bromides were subjected to nucleophilic substitution of acyl 
carbon atom using sodium benzoate with fly-ash catalyst in water medium (Scheme 2). The reaction got completed within $24 \mathrm{~h}$ and more than $60 \%$ yields of 4-substituted 1-naphthacyl benzoates (10-18) were obtained. Fly ash is a waste air-pollutant and it has many chemical components[3, 41-43] such as $\mathrm{SiO}_{2}, \mathrm{Fe}_{2} \mathrm{O}_{3}, \mathrm{Al}_{2} \mathrm{O}_{3}, \mathrm{CaO}, \mathrm{MgO}$ and insoluble residues. During the course of the reaction these species exhibit their catalytic effects in the bromination in aryl methyl group and nucleophilic substitution at acyl carbon of acyl bromides in the side chain. After separating the products, the catalyst was recycled for further reactions. Unfortunately, the catalyst was ineffective for further reaction cycle.

\subsection{Hammett spectral correlation analysis}

The study of Hammett spectral correlation analysis was useful for the prediction of ground state molecular equilibration [44] of organic substrates such as $s$-cis and s-trans conformers of unsaturated systems, acyl halides, haloketones and acyl esters. Their use in structure parameter correlations and structure-activity relationships were important for studying biological activities [45] normal co-ordinate analysis [46, 47] and reaction mechanisms [48] through transition state.

Many organic compounds are identified by the direct analysis of their infrared spectra with authentic samples. The importance of infrared spectroscopy in material sciences is to provide the information about the nature, concentration and structure of samples at the molecular levels [50]. Infrared spectroscopy is a useful technique for the qualitative and quantitative study of natural and synthetic molecules [49]. McBee and Christman et. al. [51] have investigated the effect of $\alpha$-substituents on the carbonyl group stretching frequency in the infrared spectra of halogenated acetic esters. Rasmussen and Brattain [52] have studied the infrared spectra of a number of aliphatic esters and noted that an electron-donor group causes a shift of the carbonyl group stretching band towards longer wavelength; an electron withdrawing group causes the reverse effect. Generally, the carbonyl stretching frequencies of the halo aryl esters are higher than those of the non-halo aryl esters. It was expected that fluorine as the $\alpha$-substituent would cause a marked shift and recent studies [53] of fluorine substituted aryl esters have substantiated this expectation Introduction of an $\alpha$-halogen atom into any open chain structure, $-\mathrm{CH}_{2}-\mathrm{CO}-$, results in increase of carbonyl group frequency. As rotation is possible about the $\mathrm{C}-\mathrm{C}-$ bond, one would expect infinite number of conformations in $\alpha$-haloketones. One would expect a second carbonyl absorption arising 
from a rotational isomer in which the chlorine atom is twisted away from the oxygen and the carbonyl frequencies should therefore be unaffected. Hence, compounds such as bromoacetic and fluoroacetic esters are clearly marked and they do in fact exhibit a second $\mathrm{C}=\mathrm{O}$ absorption in the solution state which is very close to the carbonyl frequencies of acetic acid itself. The halo acylacetophenone[54] have been shown to exhibit rotational isomerism which gives raise to two carbonyl frequencies and the higher of these has been assigned to the form in which the halogen atom is cis with respect to the carbonyl oxygen and therefore nearer to it in space. Dipolemoment [54] studies established that the more polar cis-configuration is the stable form under these conditions in haloacetone. The configurational isomers of the halo acylacetophenones have been recognized through the intensities of the individual carbonyl bonds in their spectra. Dipole moment and spectroscopic studies have shown that the more polar cis- form (Fig. 1) is more stable in liquid and solid states whereas the gauche form (Fig. 2.) is favoured in vapour state. These findings are clear evidence for the presence of genuine rotational isomers. Moreover, the same trend was observed in the present study with naphthyl systems.

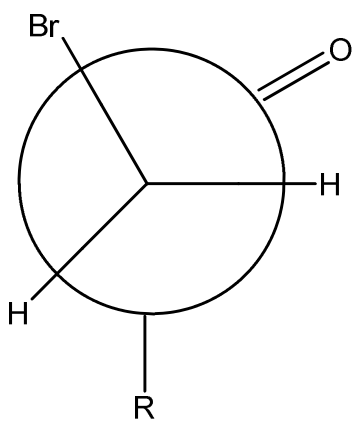

Fig. 1.

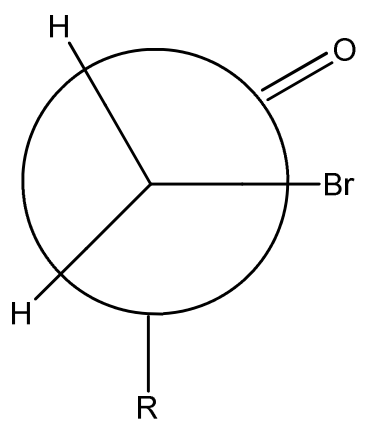

Fig. 2.

A large number of spectral data relating to substituted styryl naphthyl ketones accumulated in the previous investigations were correlated using a variety of LFER models, conventionally used for the study of structure reactivity and structure-property relationships. It was assumed that it should be possible to find an adequate approach to study the transmission of substituent effects in the multi-substituted ketones. Generally attempts have been made to use simple Hammett Equation [55] as shown in (eq. 1a). However Hammett-Taft (Extended Hammett Equation) DSP model, (eq. 1b) and Swain-Lupton's (eq. 1c) [56] were also used frequently in their general form $[57,58]$. 


$$
\begin{array}{lc}
\text { a. } & s=\rho \sigma+s_{o} \\
\text { b. } & s=\rho_{I} \sigma_{I}+\rho_{R} \sigma_{R}+s_{o} \\
\text { c. } & s=f F+r R+s_{o}
\end{array}
$$

In these models, the authors applied Eq. 1a and 1c only for evaluation of electronic effects in this aromatic system and $\mathrm{s}$ is the measured spectral characteristics, $\sigma_{\mathrm{m} / \mathrm{p}}, \sigma_{\mathrm{I}}, \sigma_{\mathrm{R}}, \mathrm{F}$ and $\mathrm{R}$ are substituent constants, $\rho, \rho_{\mathrm{I}}, \rho_{\mathrm{R}}, \mathrm{f}$ and $\mathrm{r}$ are the corresponding calculated proportionality constants, which, in a broad sense reflect the sensitivity of the spectral characteristics to substituent effects and $s_{o}$ is the intercept. On certain occasions, when other model failed, combined multi-parameter equations were applied, the method known to be used before and with the same precision as obtained here.

\subsubsection{Infrared spectral study}

All the synthesized 4-substituted 1-naphthacyl bromides and their esters investigated in the present study contain system which closely resemble the side chain of 21-acetoxy-20-ketosteroids. As a result of rotation about the $\mathrm{C}-\mathrm{C}$ bond, and observed IR carbonyl doublets there are mainly two pre- framed rotamers and they are shown in Fig. 3 and 4 [59].

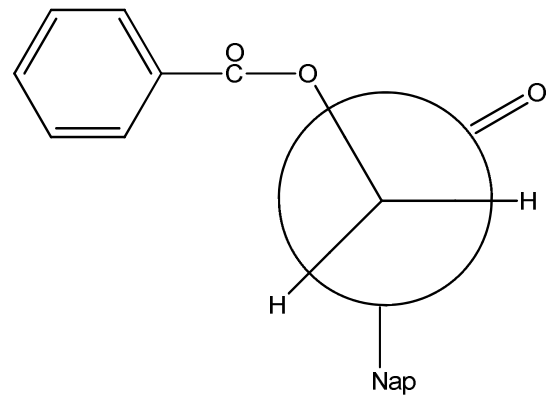

Fig. 3.

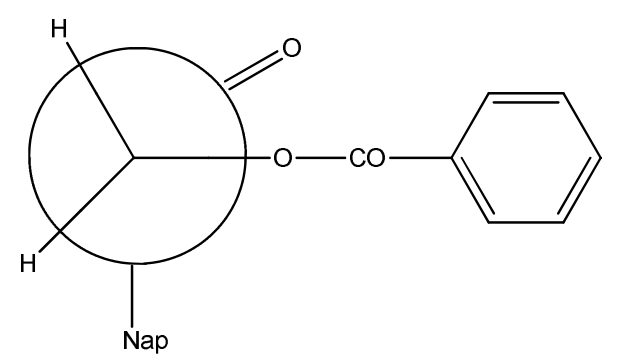

Fig. 4.

The IR spectral data of all synthesized 4-substituted 1-naphthacyl bromides are presented in Table 1. These data are correlated with Hammett substituent constants with single and multi-linear regression analysis. The results of statistical analysis are presented in Table 2. From the table 2, cis rotamer frequencies are satisfactorily correlated with Hammett $\sigma, \sigma^{+}, \sigma_{\mathrm{I}}$, and $\mathrm{F}$ parameters. The resonance effects fail in correlation in single regression analysis. The carbonyl frequencies of gauche rotamers gave satisfactory correlation coefficient with Hammett sigma constants and fail with other parameters. 


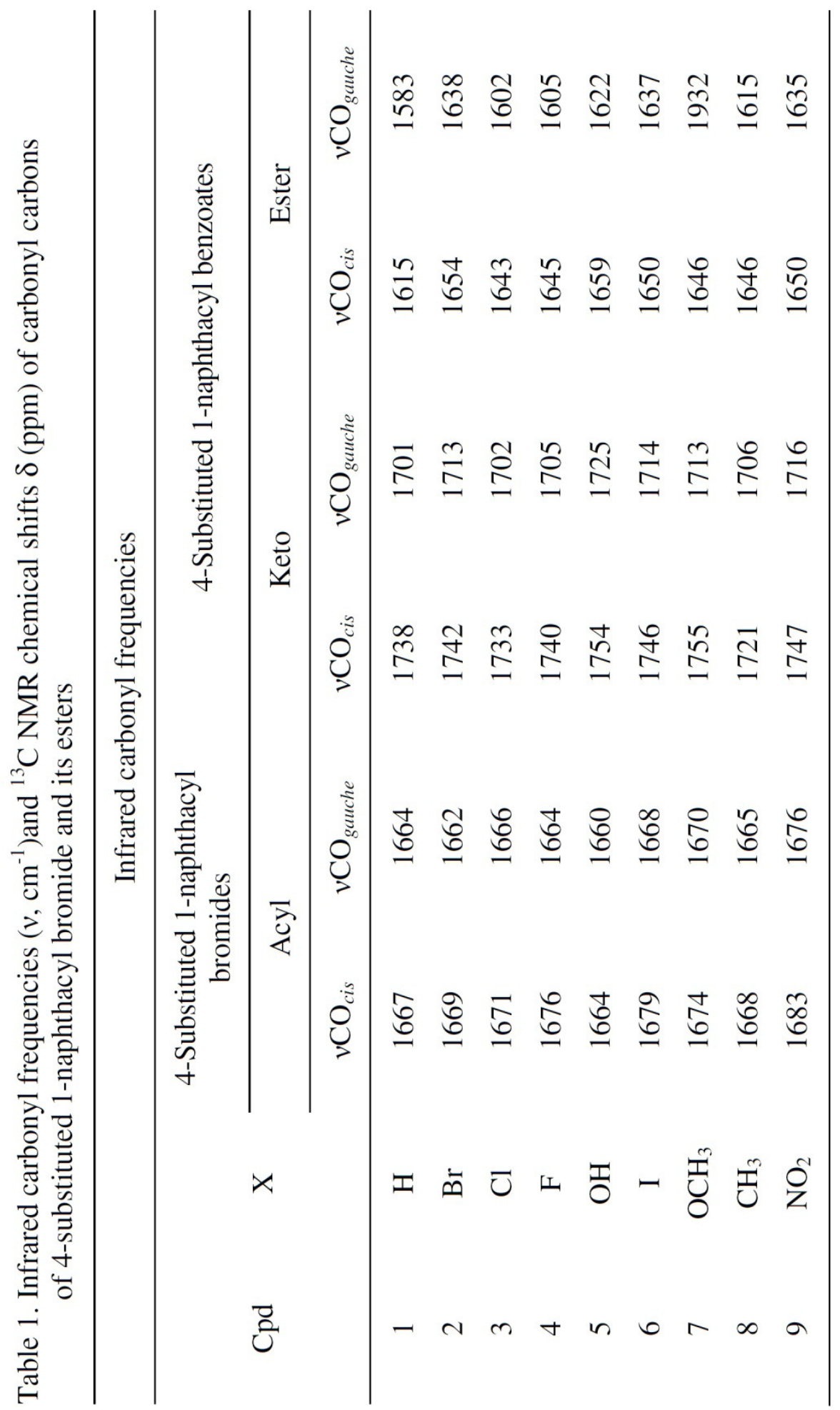




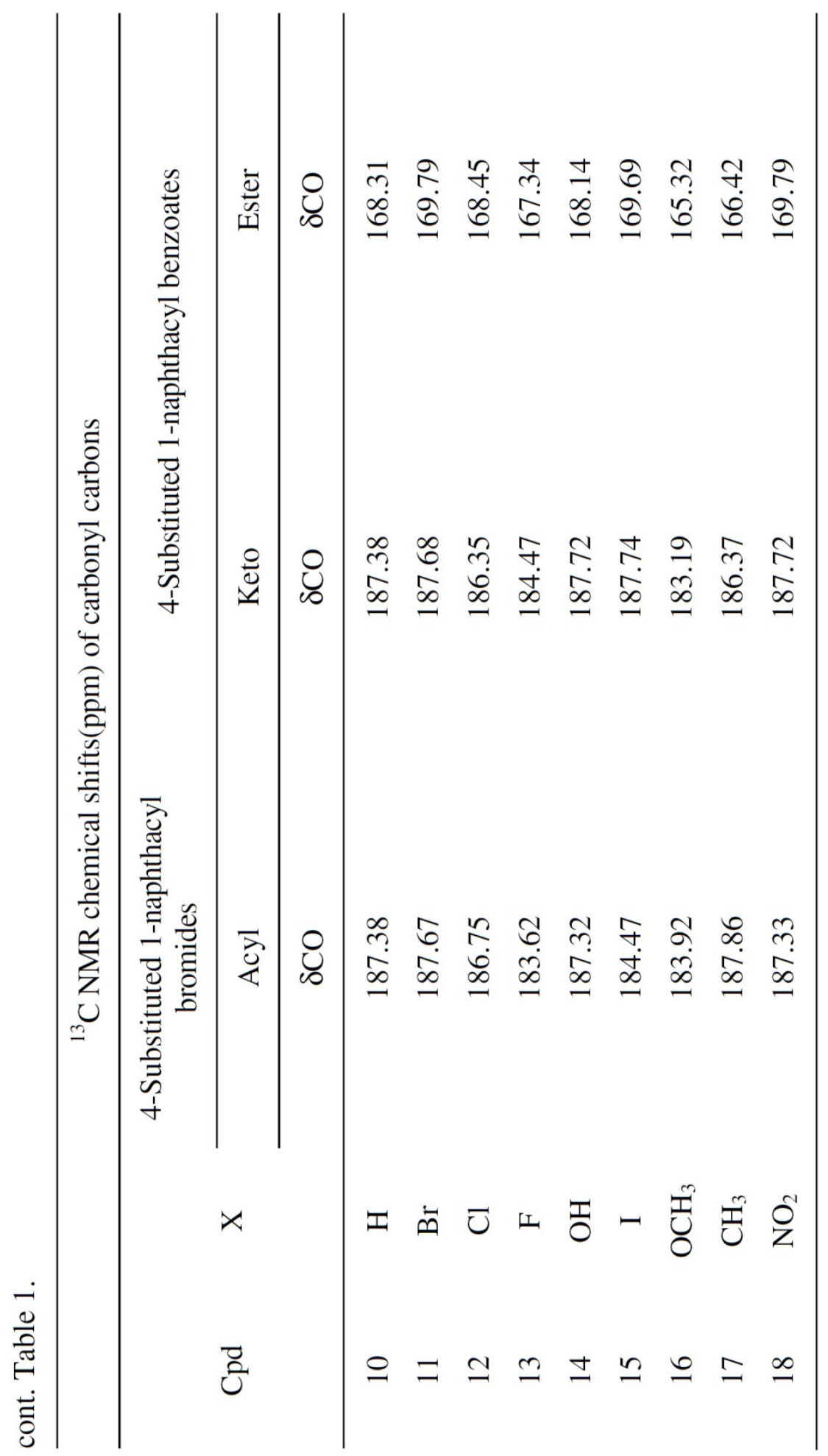




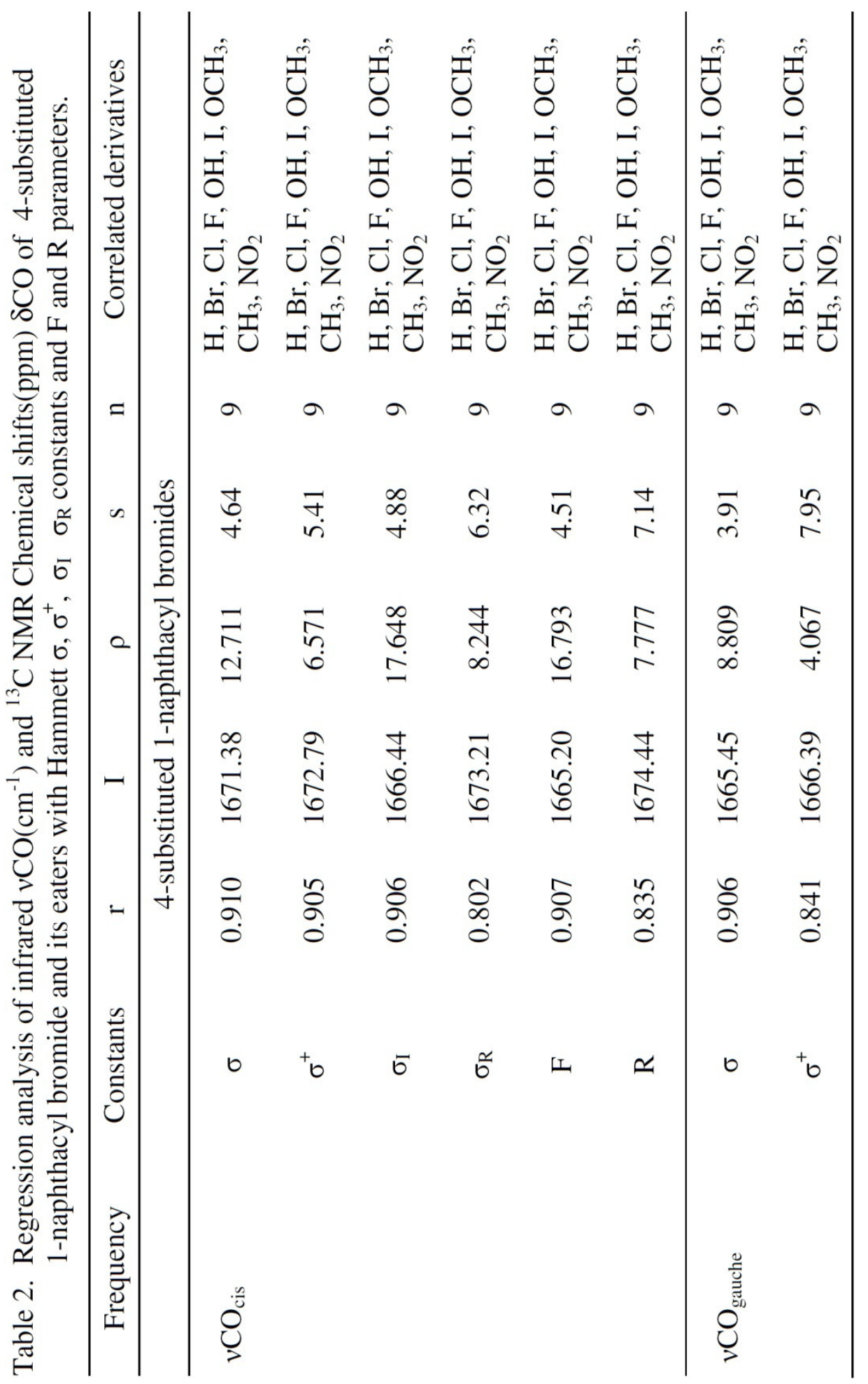




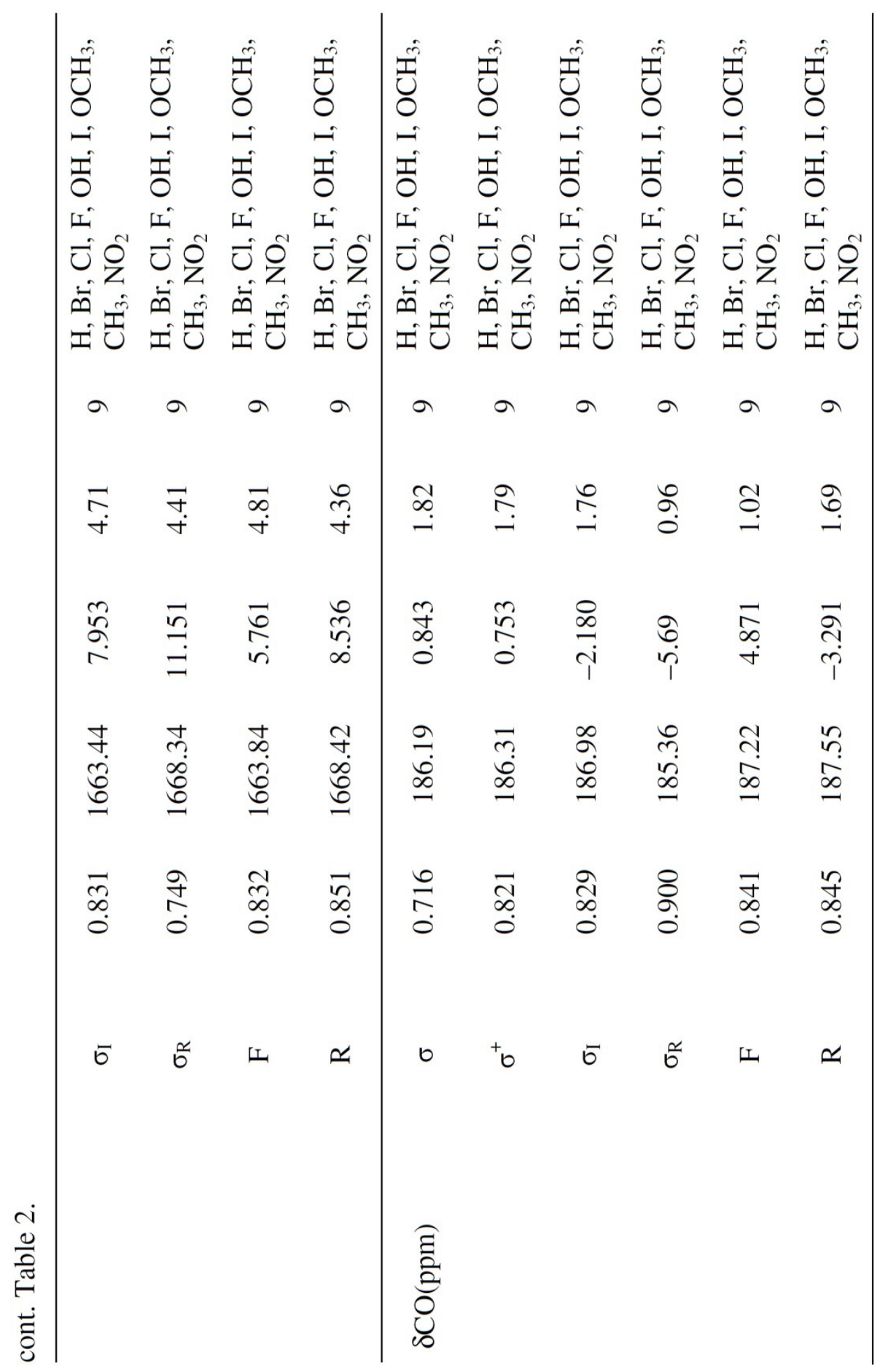




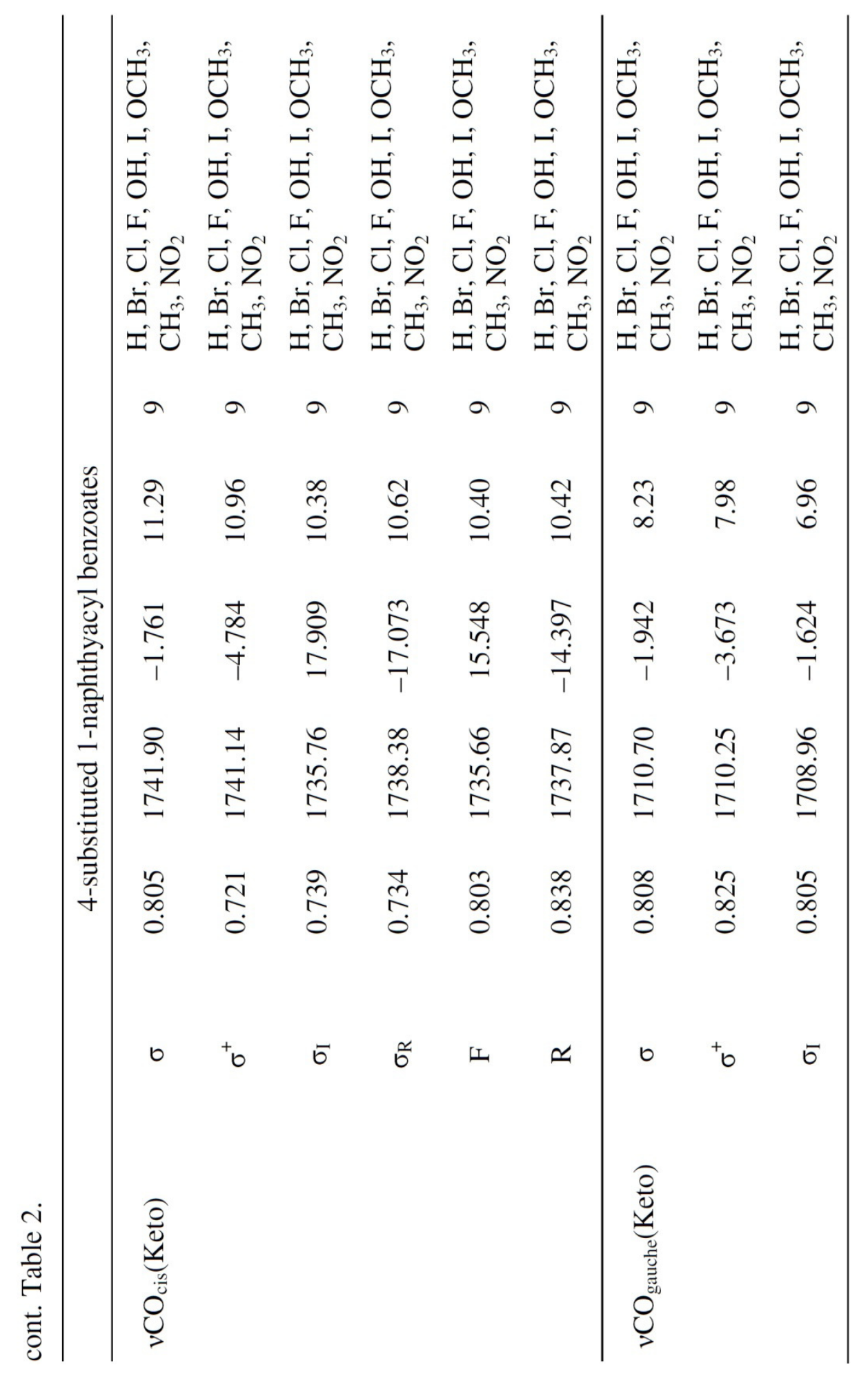




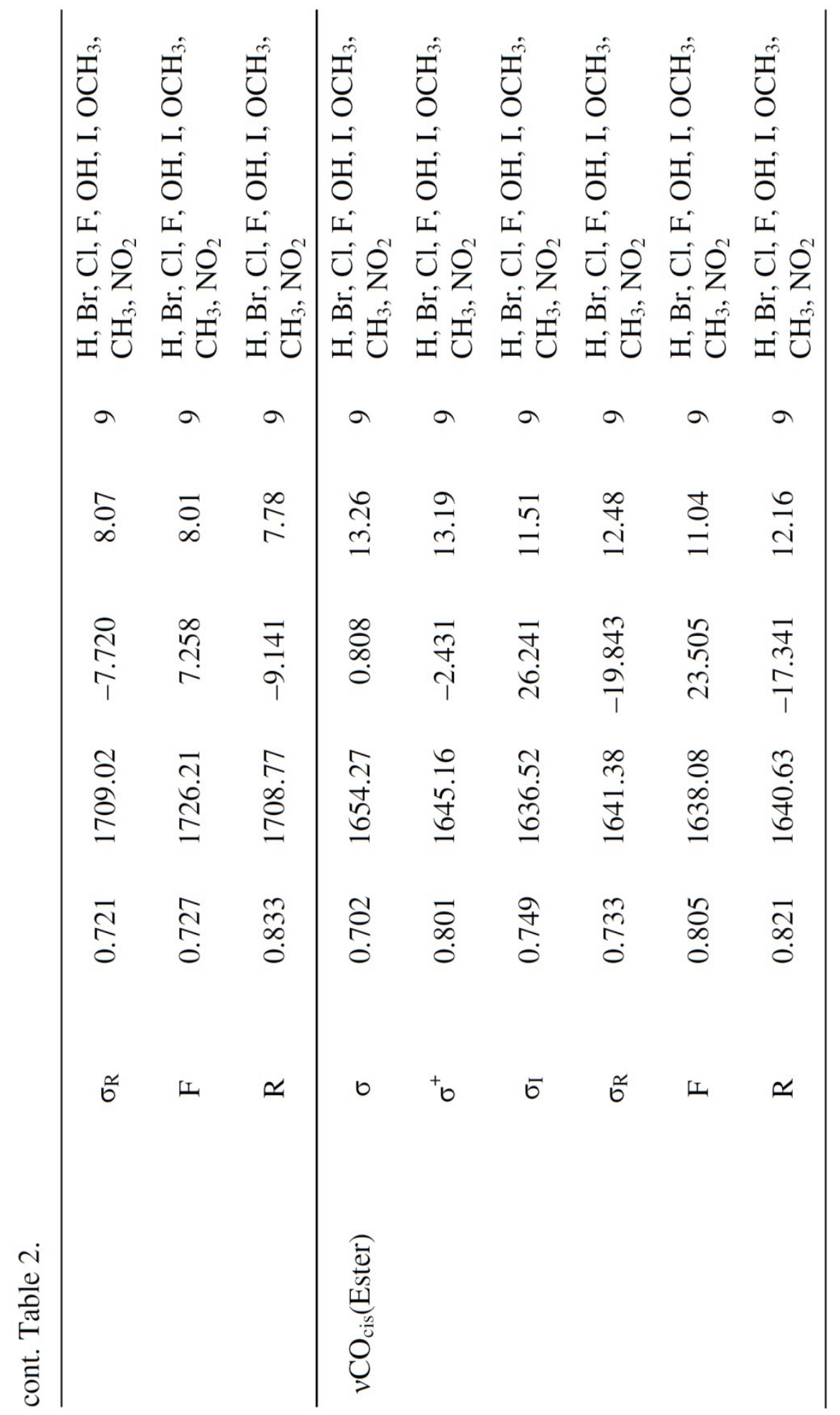




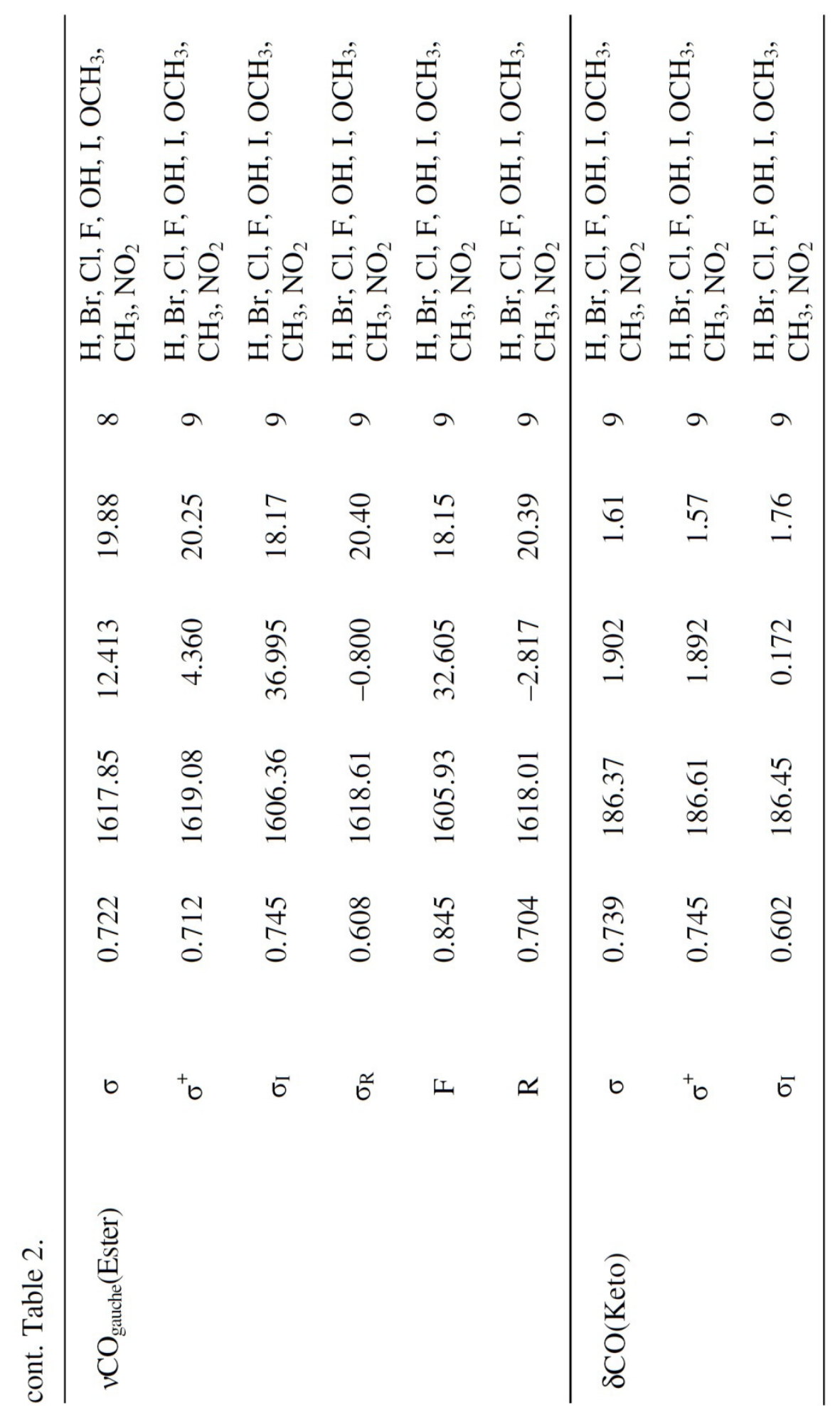




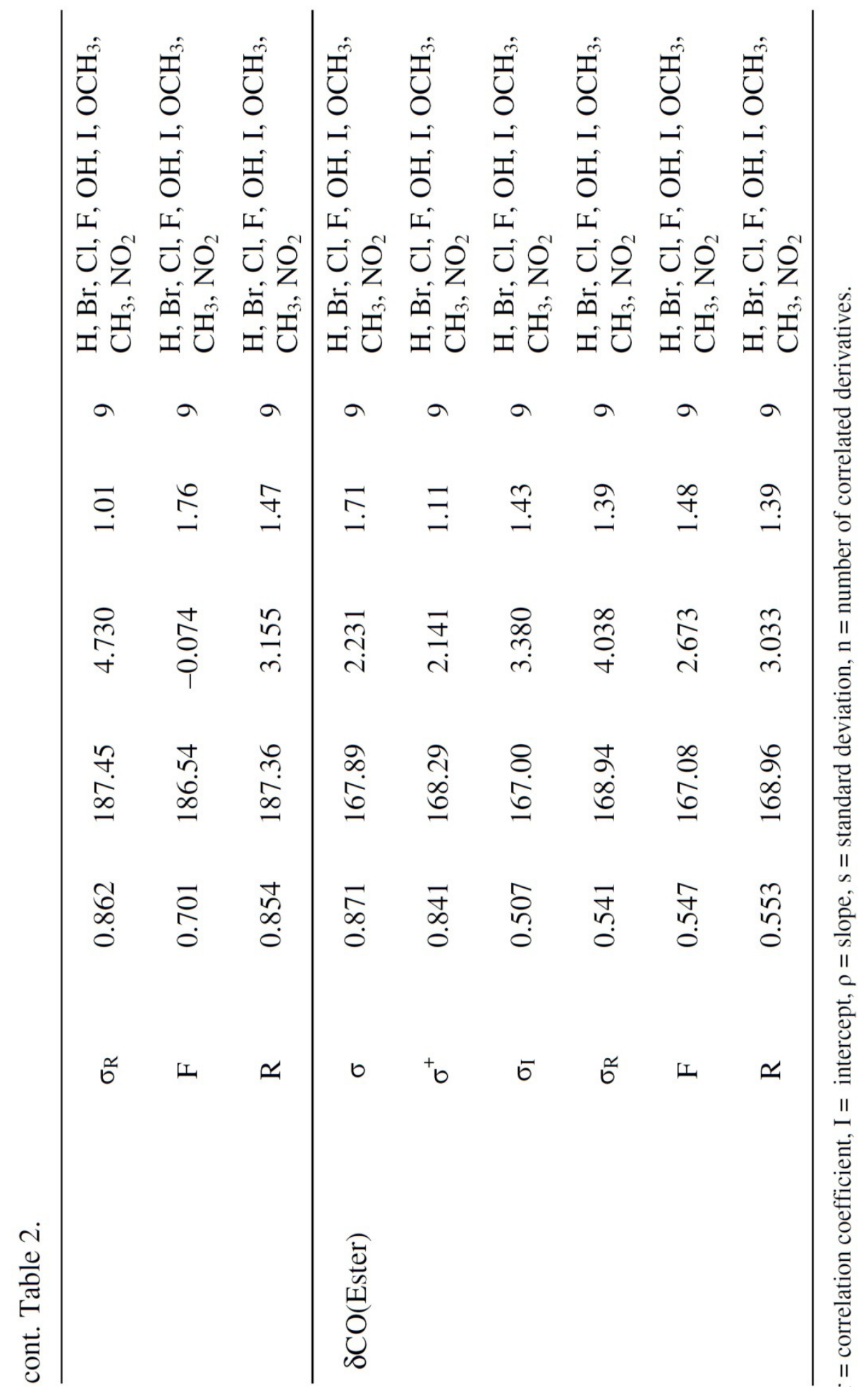


This is due to the inability and weak polar, inductive, resonance and field effects of substituents to predict the reactivity on the carbonyl group of the rotamers, through resonance as per the conjugative structure shown in Fig. 5.

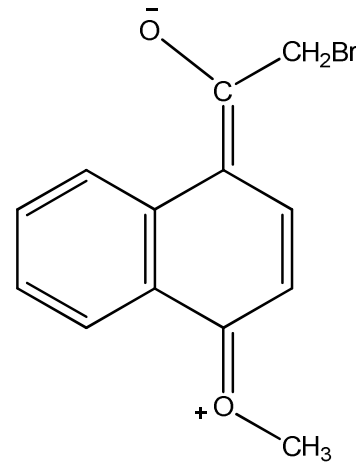

Fig. 5.

Some of the single parameter correlations of carbonyl group frequencies with Hammett substituent constants, F and R parameters were found to be poor. Therefore the author has decided to investigate the multi-regression [60-65] analysis of these carbonyl group frequencies with Swain-Lupton's [56] parameters, inductive and resonance effects of the substituents. The obtained multi-regression equations are presented in Table 3. In the multi-regression analysis, carbonyl group frequencies of both the rotamers produced satisfactory correlation coefficients collectively. The assigned carbonyl frequencies of ester and keto groups in the 4-substituted 1-naphthacyl benzoates are presented in Table 1. These frequencies are correlated with simple Hammett substituent constants, Swain Lupton's [56] constants and F and R parameters. The results of statistical analysis of these frequencies are presented in Table 2. The poor correlation obtained for the frequencies of keto $\mathrm{CO}$ cis and gauche rotamers with Hammett substituent constants, $\mathrm{F}$ and $\mathrm{R}$ parameters. This is due to the reasons stated earlier and the polar, inductive and resonance effect of the substituents completely absence from the carbonyl group and associated with the resonance conjugative structure shown in Fig. 6.

The frequencies of ester COcis and gauche of the rotamers correlated poorly with Hammett substituent constants, $\mathrm{F}$ and $\mathrm{R}$ parameters. This is due to incapability of the substituents for predicting the reactivity on the carbonyl group from naphthyl ring. Here the distance from the carbonyl group and the substituent in naphthyl ring is considered which is more than four or more carbon atom lengths. The resonance effect of the substituents is unable to predict the reactivity on not only keto- carbonyl group but also the ester carbonyl group of all compounds shown in Fig. 6 . 


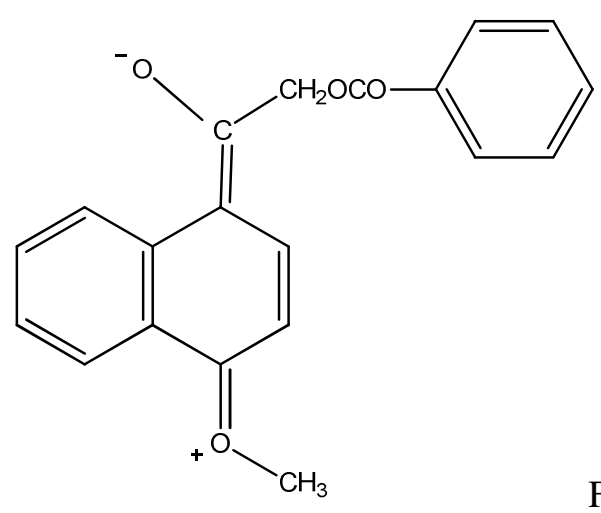

Fig. 6.

Some of the single parameter correlations fail in some cases. But these are worthwhile when seeking the multi-regression analysis of these frequencies with inductive, resonance and polar constants collectively. The data of this regression analysis are presented in Table 3.

\subsection{2. ${ }^{13} \mathrm{C}$ NMR spectral study}

From ${ }^{13} \mathrm{C}$ NMR spectra, the assigned $\delta^{13} \mathrm{C}$ carbonyl carbon chemical shifts (ppm) of synthesized 4-substituted 1-naphthacyl bromides and their esters are tabulated in Table 1. These chemical shifts are correlated separately with various Hammett substituent constants. The results of statistical analysis [59-65] of these chemical shifts are presented in Table 2. The Hammett $\sigma_{R}$ constants show only a fair degree of correlation with the chemical shifts (ppm) of carbonyl carbon of 4-substituted 1-naphthacyl bromides. The correlations of remaining Hammett constants, field and resonance effects of the substituents completely fail. This is due to the reasons stated earlier with the conjugative structure shown in Fig. 5. The $\delta^{13} \mathrm{C}$ carbonyl carbon chemical shifts of keto- and ester- moieties of 4-substituted 1-naphthyl benzoates are analyzed with Hammett simple substituent constants using single and multi-regression analysis.

The results of statistical analysis of these carbon chemical shifts are tabulated in Table 2. All simple regressions of C-13 chemical shifts of keto carbonyl carbons of these esters with Hammett substituent constants fail in correlation. This is due to the reasons stated earlier with conjugative structure shown in Fig. 6. The multi-regression analysis of these carbon chemical shifts with Swain-Lupton's[56], constants gave fair degree correlations with either $\sigma_{I}$ and $\sigma_{R}$ or $F$ and $R$ parameters shown in Table 3. 


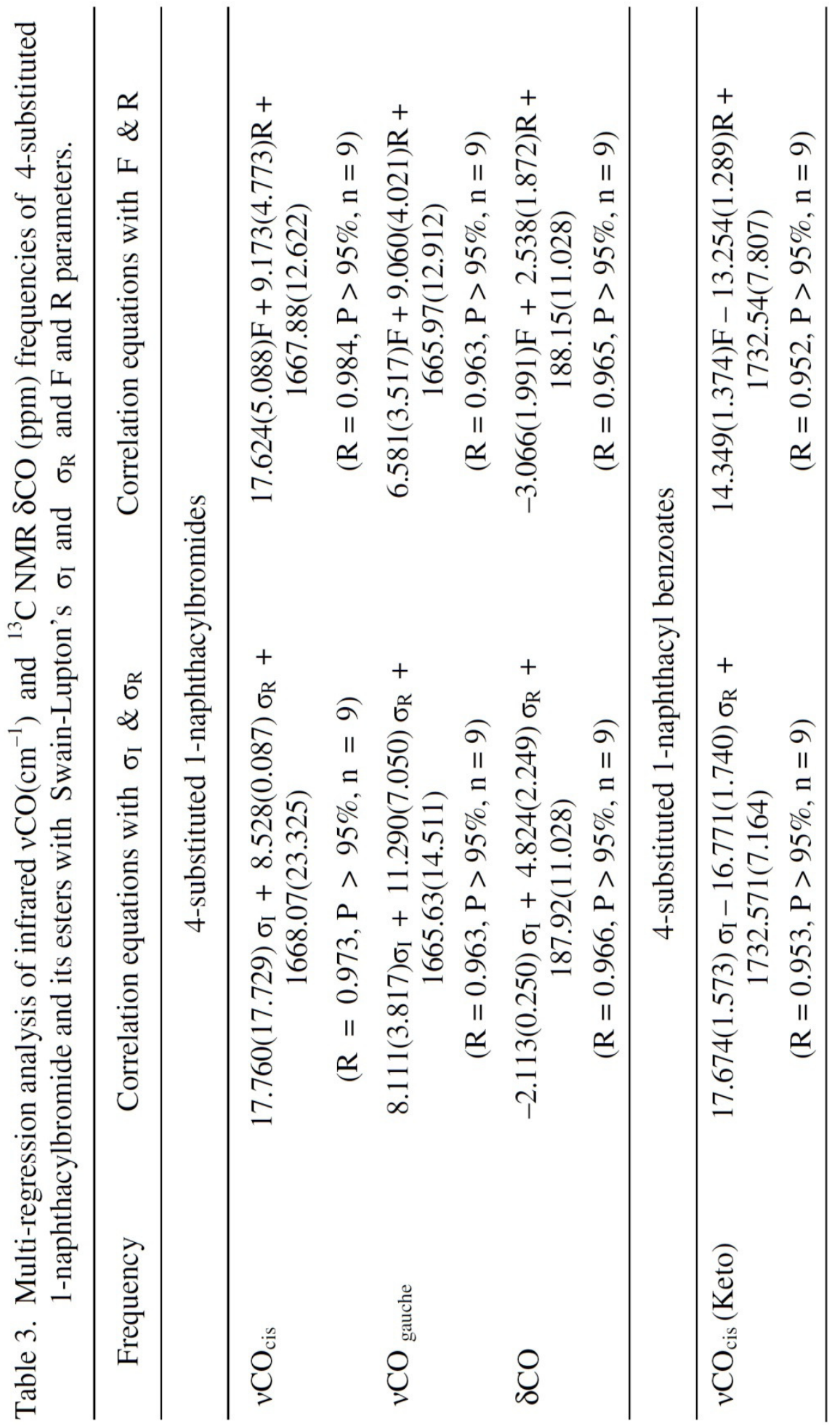




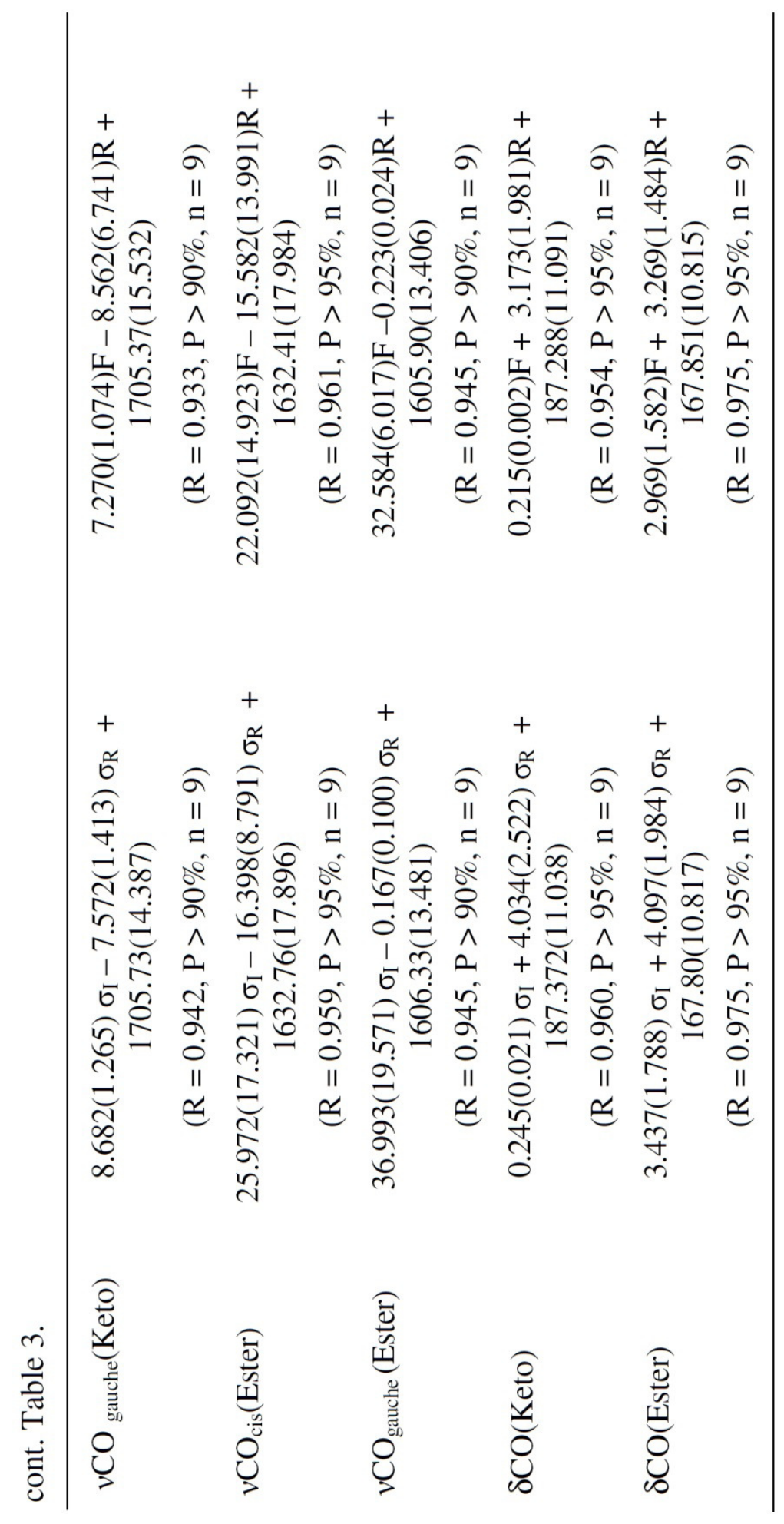




\subsection{Insect antifeedant activity}

All esters possess significant biological activities. The basic skeletons of acyl esters present widely in natural products are known to have multi-biological activity. Presence of polar functional groups, hydrophobic moiety and absence of steric hindrance near the aryl ring are attributed to the biological activities of esters and their derivatives. Many esters and their derivatives are used as antimicrobial agents [66-68], growth and enzyme activity of Aspergillus and Penicillium citrinum [69], antiviral [70], cytotoxic [71], biomass [72] and bio-pesticidal agents [73]. It is observed that only two of the synthesized esters have fair antioxidant activity.

As one of the multi-pronged activities, the insect antifeedant activity of haloketones and haloacyl compounds, has been examined. The larvae's of Achoea Janata $L$. were reared as described on the leaves of caster Riclmus Cammunls in the laboratory at the temperature range of $26 \pm 1^{\circ} \mathrm{C}$ and a relative humidity of $75-85 \%$.

The leaf-disc bioassay method $[74,75]$ was used against the 4th instar larvae to measure the antifeedant activity. The 4th instar larvae were selected for testing because the larvae at this stage feed very voraciously.

\subsubsection{Measurement of insect antifeedant activity of acyl bromide and its ester}

The diameter of $1.85 \mathrm{~cm}$ leaf discs were punched from caster leaves with the petioles intact. All acyl bromides and their esters were dissolved in acetone at a concentration of 200ppm and dipped for $5 \mathrm{~min}$. The leaf discs were air-dried and placed in 11 beaker containing little water in order to facilitate translocation of water.

Therefore the leaf discs remain fresh throughout the test period. Then acyl bromide had been sprayed on the leaf discs before placing 4th instar larvae, the test insect. The test insect was allowed to feed the leaf discs for $24 \mathrm{~h}$. The area of the leaf disc consumed has been measured by Dethler's [76] method.

The observed antifeedant activity of acyl bromides and their esters were presented in Table 4. and it reveals that the compounds 2-4 and 6 were found to reflect remarkable antifeedant among all other acyl bromide and its esters. This test was performed with the insects' on twoleaf disc already soaked under the solution of this compound. Compounds 3,4 and 6 also show enough antifeedant activity but lesser than 2. Further the compound 2 was subjected to measure the antifeedant activity at 


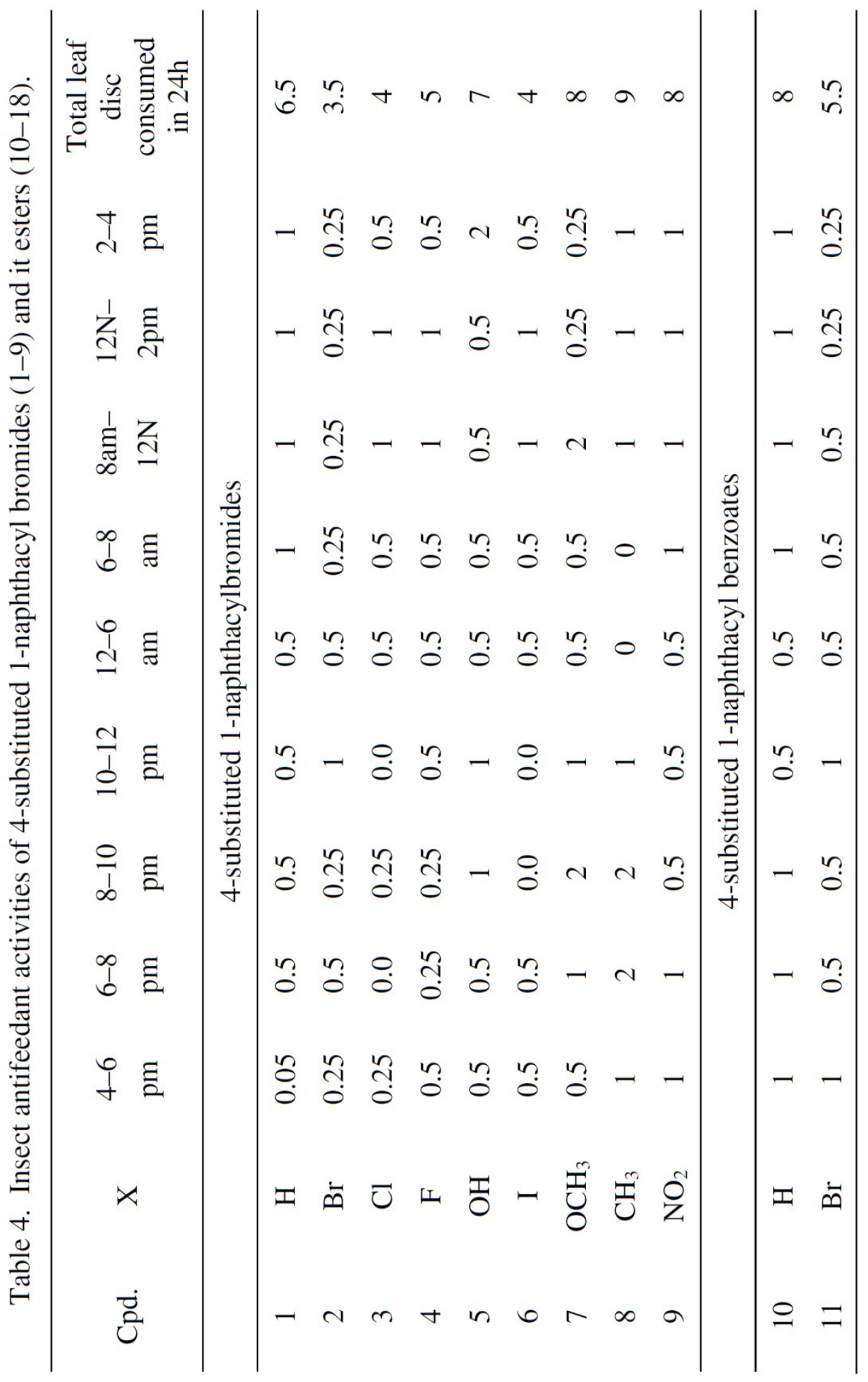




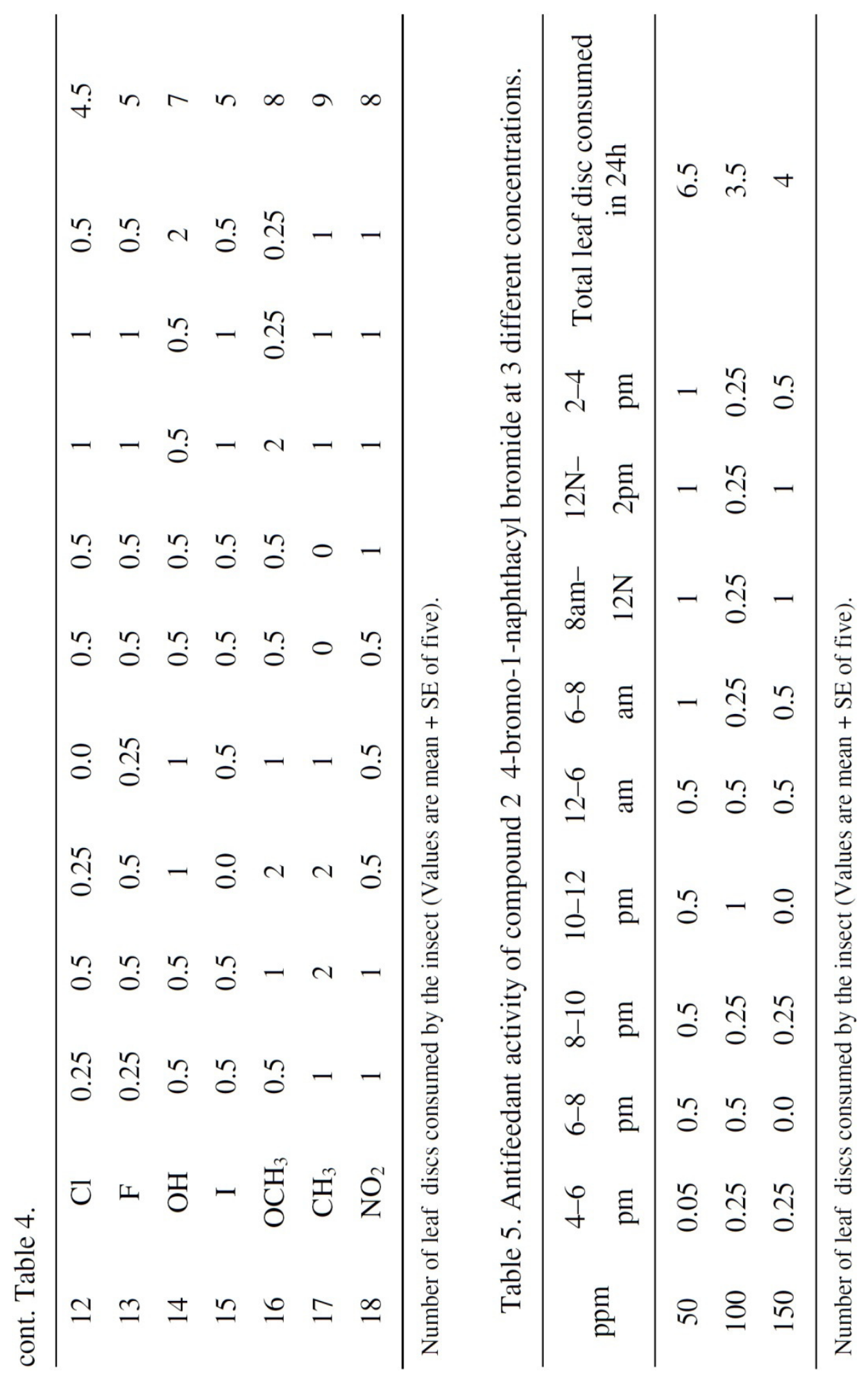


different $(50,100,150 \mathrm{ppm})$ concentrations and the observation reveals that as the concentrations decreased, the activity also decreased. It is observed from the results in Table 5 that the acyl bromide 2 4-Bromo-1naphthacyl bromide shows an appreciable antifeedant activity at 100ppm concentration.

\section{REFERENCES}

[1] G. Goel, H. P. S. Makkar, G. Francis, K. Becker, Int. J. Toxicol., 26, 279, (2007).

[2] G. Thirunarayanan, P. Mayavel, K. Thirumurthy, Spectrochim. Acta., 91A, 18, (2012).

[3] G. Thirunarayanan, G. Vanangamudi, Arkivoc., 12, 58, (2006).

[4] S. Kajigaeshi, T. Kakinami, T. Okamoto, S. Fujisaki, Bull. Chem. Soc. Jpn., 60, 1159, (1987).

[5] U. Bora, D. Dey, S. Dhar, M. K. Chaudhuri, Pure Appl. Chem., 73, 93, (2001).

[6] M.K. Chaudhuri, A.T. Khan, B.K. Patel, D. Dey, W. Kharma-wphlang, T.R. Lakshmiprapha, G.C. Mandal, Tetrahedron Lett. 39, 8163, (1998).

[7] H. Zhao, Z. Song, J.V. Cowins, O. Olubajo, Int. J. Mol. Sci., 9, 33, (2008).

[8] C.T. Chen, Y.S. Munot, J. Org. Chem., 70, 8625, (2005).

[9] J.D. Lou, Z.N. Xu, Tetrahedron Lett., 43, 6149,(2002).

[10] B. Kalluraya, G. Ray, Indian J. Chem., 42(B), 2556, (2003).

[11] G. Thirunarayanan, M. Gopalakrishnan, G. Vanangamudi, Spectrochim. Acta. 67A, 1106, (2007).

[12] F.F. Bamoharram, M.M. Heravi, M. Roshani, M. Jahangir, A. Gharib, J. Mol. Catal., 271A, 126, (2007).

[13] S. Adimurthy, G. Ramachandraiah, A.V. Bedekar, S. Ghosh, B.C. Ranu, P.K. Gosh, Green Chem., 8, 916,(2006).

[14] H. Sharghi, M. Hosseini Sarvari, Tetrahedron., 59, 3627, (2003).

[15] B. D. Paul, C. Dreka, J. L. Summers, M. L. Smith, J. Anal. Toxicol., 20, 506, (1996).

[16] J. Deli, T. Loand, D. Szabo, A. Foldsi, Pharmazie., 39, 539, (1984).

[17] L. Sun, S. Zheng, J. Wang, X. Shen, Indian J. Chem., 41B, 1319, (2002).

[18] M.G. Ahmed, S.A. Ahmed, U.K.R. Romman, T. Sultana, M.A. Hena, S. Kiyooka, Indian J. Chem., 41B, 368, (2002).

[19] L.Q. Wu, C.G. Yang, Y.F. Wu, L.M. Yang, J. Chin. Chem. Soc., 56, 606, (2009). 
[20] L.C. King, G.K. Ostrum, J. Org. Chem., 29, 3459, (1964).

[21] P.A. Levene, Org. Synth., II, 88, (1943).

[22] L.A. Yanovskaya, P.A., Terentev, L.N. Belenkii, J. Gen. Chem., 22, 594, (1952).

[23] F. Krohnke, K. Ellegast, Chem. Ber., 86, 1556 (1953).

[24] E.I. Sanches, M.J. Fumarola, J. Org. Chem., 47,1588, (1982).

[25] A.N. Mayeno, A.J. Curran, R.L. Roberts, C.S. Foote, J. Biol. Chem., 264, 5660 (1989).

[26] D.I. Cajetan, T. Bekele, S. France, J. Wolfer, A. Weatherwax, A.E. Taggi, T. Lectka, J. Org. Chem., 71, 8946, (2006).

[27] R.E. Lovins, L.J. Anrews, R.W. Keef, J. Org. Chem., 28, 2847, (1963).

[28] N.E. Starostenko, N.O. Adeeva, F.N. Zeiberlikh, L.N. Kurkovskaya, N.N. Suvorov, Chem. Heterocycl. Compd., 23, 271, (1987).

[29] S.B. Patwari, M.A. Baseer, Y.B. Vibhute, S.R. Bhusare, Tetrhedron Lett., 44, 4893, (2003).

[30] K. Wakasugi, A. Nakamura, Y. Tanabe, Tetrahedron Lett., 42, 7427, (2001).

[31] D. Taber, J. Am. Chem. Soc., 128, 13052, (2006).

[32] W.J. Yoo, C.J. Li, J. Org. Chem., 71, 6266, (2006).

[33] R.V.H. Jones, W.E. Lindsell, D.D. Palmer, P.N. Preston, A.J. Whitton, Tetrahedron Lett., 46, 8695, (2005).

[34] M. Pittelkow, F.S. Kamounah, U. Boas, B. Pedersen, J. B. Christensen, Synthesis., 2485, (2004)

[35] H. Merima, W. Gunnar, Cellulose., 14, 347, (2007).

[36] Y. Halpern, US Patent No. 5035840, (1991).

[37] R. Moumne, S. Lavielle, P. Karoyan, J. Org. Chem., 71, 3332, (2006).

[38] W.W. Christie, Advances in Lipid Methodology II, (Oily Press, Dundee), (1993), pp.69-111.

[39] P. Ananthakrishna Nadar, C. Gnanasekaran, Indian J. Chem., 14B, 606, (1976).

[40] G. Thirunarayanan, G. Vanangamudi, V. Sathiyendiran, K. Ravi, Indian J. Chem., 50B, 593, (2011).

[41] M. Gopalakrishnan, P. Sureshkumar, V. Kanagarajan, J. Thanusu, Catal. Commun., 6, 753, (2005).

[42] M. Gopalakrishnan, P. Sureshkumar, V. Kanagarajan, J. Thanusu, R. Govindaraju, Arkivoc., 13, 130, (2006).

[43] M. Gopalakrishnan, P. Sureshkumar, V. Kanagarajan, J. Korean Chem. Soc., 51(6), 520, (2007).

[44] A. Perjessy, M. Lacucova, Coll. Czech. Chem. Commun., 36, 2944, (1971). 
[45] C.M. Devia, N.B. Pappano, N.B. Debasttista, Rev. Microbiol., 29, 307, (1998).

[46] V. Krishnakumar, R. Ramasamy, Indian J. Pure Appl. Phys., 40, 252, (2002).

[47] A. Sharma, V.P. Gupta, A. Virdi, Indian J. Pure and App. Phys., 40, 46, (2002).

[48] G.K. Dass, Indian J. Chem., 23A, 23, (2001).

[49] C. Pellerin, I. Pelletier, Lab International Plus., 19, 10, (2005).

[50] P.R. Griffiths, J.M. Chalmers, (2003), Handbook of Vibrational Spectroscopy, (John Wiley \& Sons Inc., Chinchester, vol. 4, p.2756.

[51] E.T. McBee, D.L. Christman, J. Am. Chem. Soc., 77, 755, (1955).

[52] R.S. Rasmussen, R.R. Brattain, J. Am. Chem. Soc., 71, 1073, (1949).

[53] M. Hauptdchein, C.S. Stokes, E.A. Nodiff, J. Am. Chem. Soc., 74, 4005, (1952).

[54] L.J. Bellamy, R.L. Williams, J. Chem. Soc., 4297, (1957).

[55] J. Shorter, Correlation analysis in Chemistry: An introduction to linear free energy relationships, Clarendon Press, London, (1973).

[56] C.G. Swain, E.C. Lupton, Jr., J. Am. Chem. Soc., 90, 4328, (1968).

[57] M. Mišić-Vuković, M. Radojković-Veličković, J. Serb. Chem. Soc. Rev., 63, 585, (1998)

[58] S. Jovanovic, D. Mijin, M.M. Vuković, Arkivoc., 10, 116, (2006).

[59] P. Ananthakrishna Nadar, C. Gnanasekaran, Indian J. Chem., 17B, 391, (1979).

[60] S.R. Annapoorna, M. Prasad Rao, B. Sethuram, Indian J. Chem., 41A, 1341,(2002).

[61] G. Thirunarayanan, P. Ananthakrishna Nadar, J. Indian Chem. Soc., 83, 1107 (2006).

[62] G. Thirunarayanan, P. Ananthakrishna Nadar, J. Korean Chem. Soc., 50, 183, (2006).

[63] R. Suresh, D. Kamalakkannan, D. Ranganathan, R. Arulkumaran, S.P. Sakthinathan, S. Vijayakumar, K. Sathiyamoorthi, V. Mala, G. Vanangamudi, K. Thirumurthy, P. Mayavel, G. Thirunarayanan, Spectrochim. Acta. 101A, 239, (2013).

[64] G. Thirunarayanan, Indian J. Chem., 46B, 1511, (2007).

[65] P. Janaki, K. G. Sekar, G. Thirunarayanan, J. Saudi Chem. Soc., 20, 58, (2016).

[66] M. C. Unlusoy, N. Altanlar, R. Ertan, Turk. J. Chem., 29, 187, (2005).

[67] B. Ahilstrom, M. C. Bertillson, R. A. Thompson, Antimicrob. Agents Chemother., 39, 50, (1995). 
[68] A.A. Williams, E.W. Sugandhi, R.V. Macri, J.O. Falkinham, R.D. Gandour, J. Antimicrob. Chemother., 59, 451, (2007).

[69] O.E. Nwfor, M.J. Ikenebomeh, African J. Biotechnol., 8, 2843, (2009).

[70] W.B. Wan, J.R. Beadle, C. Hartline, E.R, Kern, S.L. Ciesla, N. Valiaeva, K.Y. Hostetler, Antimicrob Agents Chemother., 49, 656, (2005).

[71] B.C. Brasileiro, V.R. Pizziolo, D.S. Raslan, C.M. Jamal, D. Silveira, Brazilian J. Pharm. Sci., 42, 195, (2006).

[72] A. Konopka, T. Zakharova, M. Bischoff, L. Oliver, C. Nakatsu, R.F. Turco, Appl. Environ. Micorbiol., 65, 3356, (1999).

[73] S. Y. Liu, F. Sporer, M. Wink, J. Jourdane, R. Henning, Y. L. Li, A. Ruppel, Trop. Med. Int. Health., 2179, (1997).

[74] G. Thirunarayanan, J. Indian Chem. Soc., 85, 447, (2008).

[75] G. Thirunarayanan, S. Surya, S. Srinivasan, G. Vanangamudi, V. Sathiyendiran, Spectrochim. Acta., 75A, 152, (2010).

[76] V.G. Dethler, (1947). Chemical insect attractants and repellants, Philadeciphia, p. 210. 